\title{
Reducing Cache Misses Through Programmable Decoders
}

\author{
CHUANJUN ZHANG \\ University of Missouri-Kansas City
}

Level-one caches normally reside on a processor's critical path, which determines clock frequency. Therefore, fast access to level-one cache is important. Direct-mapped caches exhibit faster access time, but poor hit rates, compared with same sized set-associative caches because of nonuniform accesses to the cache sets. The nonuniform accesses generate more cache misses in some sets, while other sets are underutilized. We propose to increase the decoder length and, hence, reduce the accesses to heavily used sets without dynamically detecting the cache set usage information. We increase the access to the underutilized cache sets by incorporating a replacement policy into the cache design using programmable decoders. On average, the proposed techniques achieve as low a miss rate as a traditional 4-way cache on all 26 SPEC2K benchmarks for the instruction and data caches, respectively. This translates into an average IPC improvement of 21.5 and $42.4 \%$ for SPEC2K integer and floating-point benchmarks, respectively. The B-Cache consumes $10.5 \%$ more power per access, but exhibits a $12 \%$ total memory access-related energy savings as a result of the miss rate reductions, and, hence, the reduction to applications' execution time. Compared with previous techniques that aim at reducing the miss rate of direct-mapped caches, our technique requires only one cycle to access all cache hits and has the same access time of a direct-mapped cache.

Categories and Subject Descriptors: B.3.2 [Memory Structures]: Design Styles-Cache memories

General Terms: Design, Experimentation, Performance

Additional Key Words and Phrases: Cache, low power, dynamic optimization

Extension of Conference Paper An earlier version [Zhang 2006] of this paper appears in the 33rd ACM International Symposium on Computer Architecture (ISCA2006). This paper expands on the following aspects: First, experiments have been redesigned by using SimPoints to choose the simulation points. In original work, all benchmarks were fast forwarded 2 billions instructions and execute the following 500-M instruction. Second, Section 2.2 is newly added to provide the motivation of the balance cache design. The Section 4 has been rewritten to describe the overhauled experiments. Section 5.3 is newly added to discuss the issue of subarray width and line size. The miss rate reductions and performance improvement in terms of IPC have been greatly increased because of the change of simulation points. Third, there are nine more figures and three more tables are added to the original paper. This makes the evaluation of the proposed techniques more complete.

Authors' address: Chuanjun Zhang, Department of Computer Science and Electrical Engineering, University of Missouri-Kansas City, Kansas City, Missouri 64110; email: zhangchu@umkc.edu.

Permission to make digital or hard copies of part or all of this work for personal or classroom use is granted without fee provided that copies are not made or distributed for profit or direct commercial advantage and that copies show this notice on the first page or initial screen of a display along with the full citation. Copyrights for components of this work owned by others than ACM must be honored. Abstracting with credit is permitted. To copy otherwise, to republish, to post on servers, to redistribute to lists, or to use any component of this work in other works requires prior specific permission and/or a fee. Permissions may be requested from Publications Dept., ACM, Inc., 2 Penn Plaza, Suite 701, New York, NY 10121-0701 USA, fax +1 (212) 869-0481, or permissions@acm.org. (C) 2008 ACM 1544-3566/2008/01-ART24 \$5.00 DOI 10.1145/1328195.1328200 http://doi.acm.org/ $10.1145 / 1328195.1328200$

ACM Transactions on Architecture and Code Optimization, Vol. 4, No. 4, Article 24, Publication date: January 2008. 
Zhang, C. 2008. Reducing cache misses through programmable decoders. ACM Trans. Architec. Code Optim. 4, 4, Article 24 (January 2008), 31 pages. DOI = 10.1145/1328195.1328200 http://doi. acm.org/10.1145/1328195.1328200

\section{INTRODUCTION}

Increased processor clock frequency as a result of continuous transistor scaling, along with microarchitectural innovations, has led to a tremendous gap between processor and memory speed, a phenomenon known as the memory wall [Wilkes 1995; Wulf and McKee 1995]. This gap between processor and memory speed is growing at 50\% per year. General-purpose processor designers have primarily relied on cache memory hierarchies and latency-hiding techniques, such as prefetching and proper code scheduling, to reduce the performance gap. Unfortunately, with advances in technology, this memory wall has gradually moved from accessing off-chip memory to on-chip cache hierarchies, as wire delays overpass gate delays. Originally, accessing level-one (L1), level-two (L2), and off-chip memory has taken 1,10 , and 100 cycles, respectively. In the future, however, the same-sized L1 and L2 caches and memory will need around 5, 50, and 500 cycles to access, which calls for new designs to attack the prolonged memory access latencies.

The effectiveness of traditional cache designs is reaching a point of diminishing returns, especially in nanometer technologies. In a 35-nm implementation with a $10-\mathrm{GHz}$ clock frequency, accessing even a $4-\mathrm{kB} \mathrm{L} 1$ cache requires three clock cycles [Agarwal et al. 2000]. A 4-kB cache, however, is too small to provide a sufficiently low miss rate for many applications. A typical capacity of L1 caches is from 16 [Naffziger 2002] to 64-kB [Sun Microsystems 2006] for general/workstation processors, which needs five to ten cycles to access in 35-nm technology. Therefore, designing a fast access $\mathrm{L} 1$ cache with as low a miss rate as a set-associative cache is important for future processors.

Figure 1 summarizes the cache access time, power per access, area, and the average miss rate for all 26 benchmarks from SPEC2K [Standard Performance Evaluation Corporation] for both instruction and data caches for direct-mapped, $2-, 4-$, and 8-way at sizes of 8 and 16-kB. All the values are normalized to the 8-way cache. A conventional direct-mapped cache accesses only one tag array and one data array per access. A set-associative cache accesses multiple tag arrays and data arrays per access. A direct-mapped cache has the benefit of not requiring a multiplexer to combine multiple accessed data items and, therefore, can have faster access time. A direct-mapped cache is 29.5 and $19.3 \%$ faster than a same-sized 8-way cache at sizes of 8 and $16 \mathrm{kB}$, respectively.

A direct-mapped cache has the advantage of consuming less power per access, because of accessing only one way instead of multiple ways as in a set-associative cache. A direct-mapped cache consumes 74.7 and $68.8 \%$ less power than a same-sized 8-way cache at sizes of 8 and $16 \mathrm{kB}$, respectively. A direct-mapped cache is also simple to design, easy to implement, and accounts for less area. 


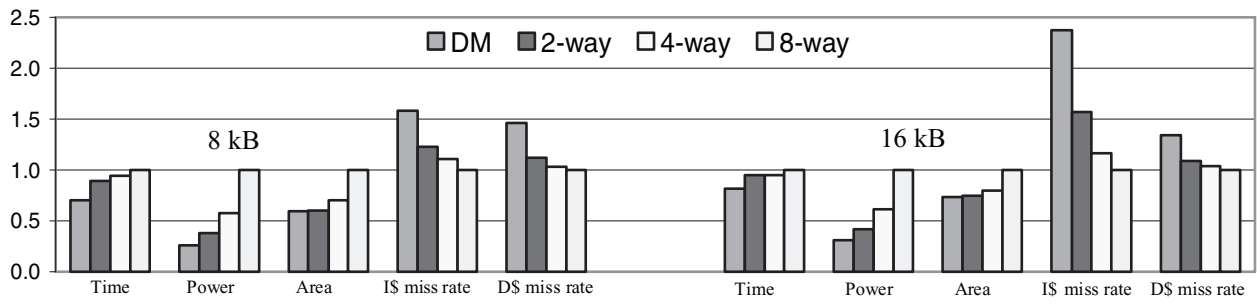

Fig. 1. Access time, power per access, area, and average miss rate of both the data cache $(D \$)$ and instruction cache $(I \$)$ at sizes of 8 and $16 \mathrm{kB}$.

A direct-mapped cache may have a higher miss rate than a set-associative cache, depending on the access pattern of the executing application, with a higher miss rate resulting in more waiting time for the next level memory accesses. On average, the miss rate of a direct-mapped instruction cache is 58 and $137 \%$ higher, while the miss rate of a direct-mapped data cache is 46 and $34 \%$ higher than a same-sized 8-way cache at sizes of 8 and $16 \mathrm{kB}$, respectively. As such, a direct-mapped cache may or may not result in better overall performance or energy usage for a particular application. Ideally, a desirable cache should have the access time of a direct-mapped cache, but with as low a miss rate as a highly associative cache.

A set-associative cache has one distinct advantage over a direct-mapped cache: cache miss reductions through replacement policy. A set-associative cache reduces the cache misses by choosing the victim from multiple cache blocks, while only one cache block can be chosen for a direct-mapped cache. Replacement policy of a set-associative cache can select a better victim through consideration of the cache access history. This is particularly true since memory accesses in a program run are extremely unbalanced, causing some cache sets to be accessed heavily, while others remain underutilized. Accessing a setassociative cache, however, requires longer access time and more power with a larger cache area.

We propose a novel mechanism to provide the benefits of cache block replacement while maintaining the constant access time of a direct-mapped cache. We call this the balanced cache, or simply, the B-Cache. The major contributions of our work are as follows:

(1) We propose to increase the decoder length of a traditional direct-mapped cache by three bits. For example, a $7 \times 128$ (7-bit addresses by 128 outputs) decoder is required for a traditional direct-mapped cache with 128 sets. For the proposed $B$-Cache, we use a $10 \times 128$ decoder. This creates the following effects: (a) Accesses to heavily used sets can be potentially reduced to one eighth $\left(1 / 2^{(10-7)}\right)$ of the original design; therefore, we avoid not only dynamically detecting these heavily used sets, but we also eliminate the corresponding hardware overhead, and (b) only one-eighth of the memory address space has mapping to the cache sets. We call this the limited-memory address mapping. 
(2) We propose to incorporate a replacement policy to the $B$-Cache. For a cache miss, when the desired address cannot find mapping to the cache sets because of the above-mentioned limited address mapping, the B-Cache can increase the accesses to the underutilized sets through the replacement policy without explicitly dynamically detecting these underutilized sets.

(3) We propose to use a programmable decoder for the B-Cache, since the $B$-Cache would dynamically determine which memory address has a mapping to the cache sets after a cache miss because of the limited memory address mapping.

Using execution driven simulations, we demonstrate that the B-Cache achieves an average miss rate reduction of 54.1 and $23.5 \%$ on all 26 benchmarks from the SPEC2K suite for L1 direct-mapped $16-\mathrm{kB}$ instruction and data caches, respectively. This translates into an instruction per cycle (IPC) improvement of up to $330 \%$ and an average IPC improvement of 21.5 and $42.4 \%$ for SPEC2K integer and floating-point benchmarks, respectively. Although the B-Cache consumes $10.5 \%$ more power per access than the original direct-mapped cache, the $B$-Cache achieves $12 \%$ a total memory access-related energy savings as a result of the miss rate reductions and, hence, a reduction in the application's execution time. Furthermore, compared with other techniques that reduce a direct-mapped cache's conflict misses, the access time of the B-Cache is the same as that of a traditional direct-mapped cache. Last, the $B$-Cache requires only one cycle to access all cache hits, while other techniques either need a second cycle to access part of the cache hits or must have a longer access time than a direct-mapped cache.

Section 2 further motivates the proposed technique. Section 3 describes the organization of the B-Cache. Experimental methodology and results are presented in Section 4. Section 5 describes the programmable decoder design. Performance and energy analysis are presented in Section 6. Related work is discussed in Section 7 and concluding remarks are given in section 8.

\section{MOTIVATION}

In this section, we introduce the problem and then examine the cache sets usage during a program's execution. We demonstrate our solution through a simple example.

\subsection{The Problem}

Memory reference addresses are mapped to cache sets based on index decoding. Because of the well-known locality [Kharbutli et al. 2004; Peir et al. 1998] exhibited in both instruction and data, some cache sets are accessed more frequently than others and thus generate more cache misses, while other cache sets are underutilized. Substantial research effort has been conducted to reduce the cache misses for direct-mapped caches. A victim buffer [Jouppi 1990] is a small fully-associative cache that can resolve cache misses for small direct-mapped caches. An extra cycle is required to access the victim buffer when the directmapped cache and the buffer are accessed sequentially or the access time of the 


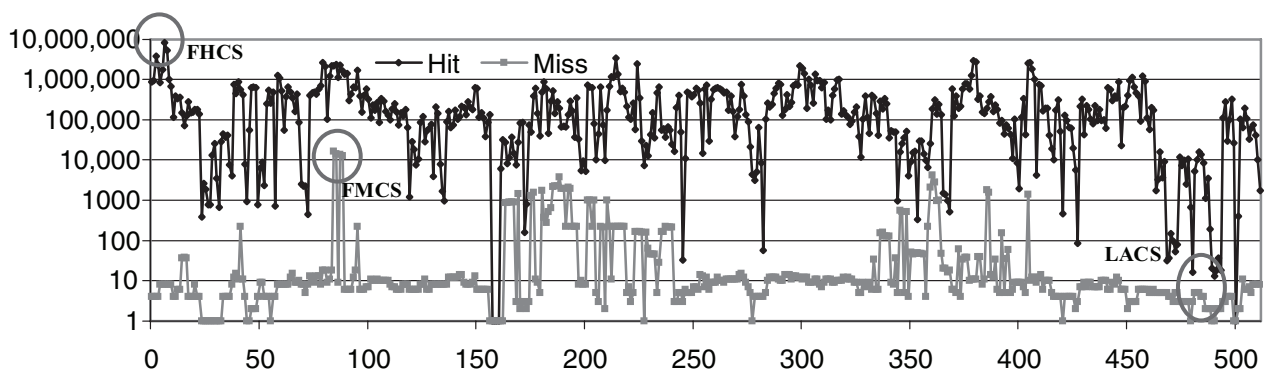

Fig. 2. Instruction cache hits and misses on each cache set of benchmark parser.

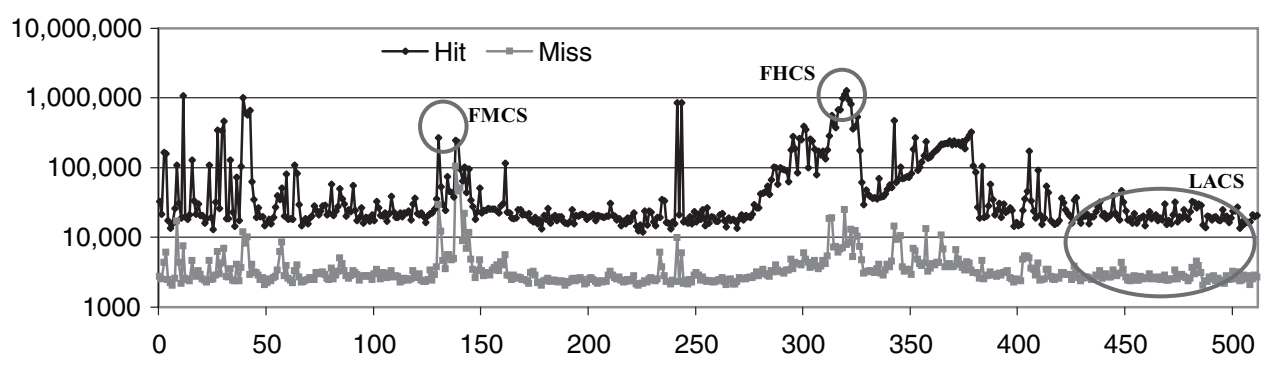

Fig. 3. Data cache hits and misses on each cache set of benchmark parser.

cache could be prolonged if the caches are checked concurrently. A multiplexer would be required to select the desired output from the buffer or the cache. The column associative cache [Agarwal and Pudar 1993], the adaptive group associative cache [Peir et al. 1998], the predictive sequential associative cache [IBM 3081; Calder et al. 1996], and the partial address matching cache [Liu 1994] trade varied hit latencies for reduced access time of set-associative caches. Multiple hit latencies disrupt data-path pipelines and complicate the design of pipelined caches [Agarwal et al. 2003].

\subsection{Cache Access Behavior Analysis}

Figures 2 and 3 show the cache hit and miss numbers on each set of instruction and data caches of the benchmark parser from SPEC2K, respectively. The cache is configured as a direct-mapped at size of $16 \mathrm{kB}$ and a line size of 32 bytes. We categorize the cache access pattern into three types: frequent hit sets, frequent missed sets, and less accessed sets. We classify a set as either a frequent hit set or a frequent miss set when the cache's hits or misses happen in a set that is two times higher than the average. We classify a set as a less accessed set when the total number of accesses to a set is less than one-half of the average. The frequent hit sets have many more hits (such as the sets of $6,7,59,214$, 224 , and 380 for instruction cache and the sets of $11,39,241,243,319,320$, and 321 for data cache) than other sets. The cache misses occur more often on a frequently missed set, such as the sets of $84,85,87$, and 88 for the instruction cache and the sets of 138,139 , and 140 for the data cache. The cache misses occurring on these cache sets, as shown in Table I, account for around 90 and 
Table I. Instruction and Data Cache Memory Access Behavior of Benchmark Parser ${ }^{a}$

\begin{tabular}{|l|c|c|c|c|c|c|c|}
\hline \multicolumn{7}{|c|}{ Instruction Cache } \\
\hline Associativity & Missrate (\%) & fhcs (\%) & Cache hits (\%) & fmcs (\%) & Cache Misses (\%) & lacs (\%) & tca (\%) \\
\hline DM & 0.07 & 12.7 & 57.1 & 7.4 & 90.5 & 56.6 & 8.9 \\
\hline 2-Way & 0.03 & 14.1 & 48.3 & 11.3 & 87.1 & 46.1 & 10.8 \\
\hline 4-Way & 0.02 & 10.2 & 30.2 & 9.4 & 73.4 & 31.3 & 9.4 \\
\hline 8-Way & 0.01 & 9.4 & 23.2 & 15.6 & 70.8 & 17.2 & 6.5 \\
\hline \multicolumn{7}{|c|}{ Data Cache } \\
\hline Associativity & Missrate (\%) & fhcs (\%) & Cache hits (\%) & fmcs (\%) & Cache Misses (\%) & lacs (\%) & tca (\%) \\
\hline DM & 4.85 & 13.1 & 65.2 & 5.3 & 25.7 & 70.9 & 20.7 \\
\hline 2-Way & 3.44 & 10.9 & 48.2 & 2.0 & 6.6 & 57.0 & 17.4 \\
\hline 4-Way & 3.06 & 12.5 & 38.8 & 0.0 & 0.0 & 32.8 & 12.3 \\
\hline 8-Way & 2.96 & 10.9 & 24.6 & 0.0 & 0.0 & 21.9 & 9.1 \\
\hline
\end{tabular}

${ }^{a}$ The table should be read as follows, for example, at the direct-mapped instruction cache, the miss rate is $0.07 \%$; $12.7 \%$ fhcs account for the $57.1 \%$ cache hits; $90.5 \%$ of the cache misses occurs on $7.4 \%$ of the cache sets; $56.6 \%$ lacs accounts for $8.9 \%$ of the total cache accesses.

$26 \%$ of the total cache misses for the instruction and data caches, respectively. The less accessed sets have been accessed less than 9 and $21 \%$ of the total cache references, while they account for around 57 and $71 \%$ of the total capacity of the instruction and data caches, which means that the limited cache space has not been used efficiently.

From Figures 2 and 3, we conclude that if the cache sets are accessed in a balanced manner, meaning all sets are accessed more evenly, then the cache misses can be reduced significantly. Set-associative caches normally have higher hit rates than those of direct-mapped caches. To verify that balancing the accessto-cache sets would generate a higher hit rate, we measure the cache miss rates, frequently hit and missed sets, and the less accessed sets of a traditional directmapped, 2-way, 4-way, and 8-way cache, and show the results in Table I for the instruction and data caches of the benchmark parser.

From Table I, we observe that the miss rates of both the instruction and data caches are consistently reduced when associativity is increased from directmapped to 8-way. The total cache hits and misses occurred in the frequent hit and missed sets are reduced as the associativity increases. The less accessed sets are continuously reduced for instruction and data caches. This means the cache accesses are more balanced as associativity increases.

From the above observation, we can see set-associative caches have a lower miss rate, because set-associative caches can use the cache space efficiently. If we can find a way to balance the mappings of a direct-mapped cache, such that the accesses to direct-mapped cache sets are more evenly distributed to all cache sets, then we can improve the hit rate of direct-mapped caches without increasing the cache's associativity or access time.

To balance the mappings of memory reference addresses to cache sets, a straightforward method is to dynamically record the accesses to each cache set in a table. In this way, the frequent hit and missed sets and the less accessed sets can be dynamically determined, so we can remap the memory references addresses to cache sets based on the real-time information collected. The disadvantages of this method include needing extra counters or tables to record 

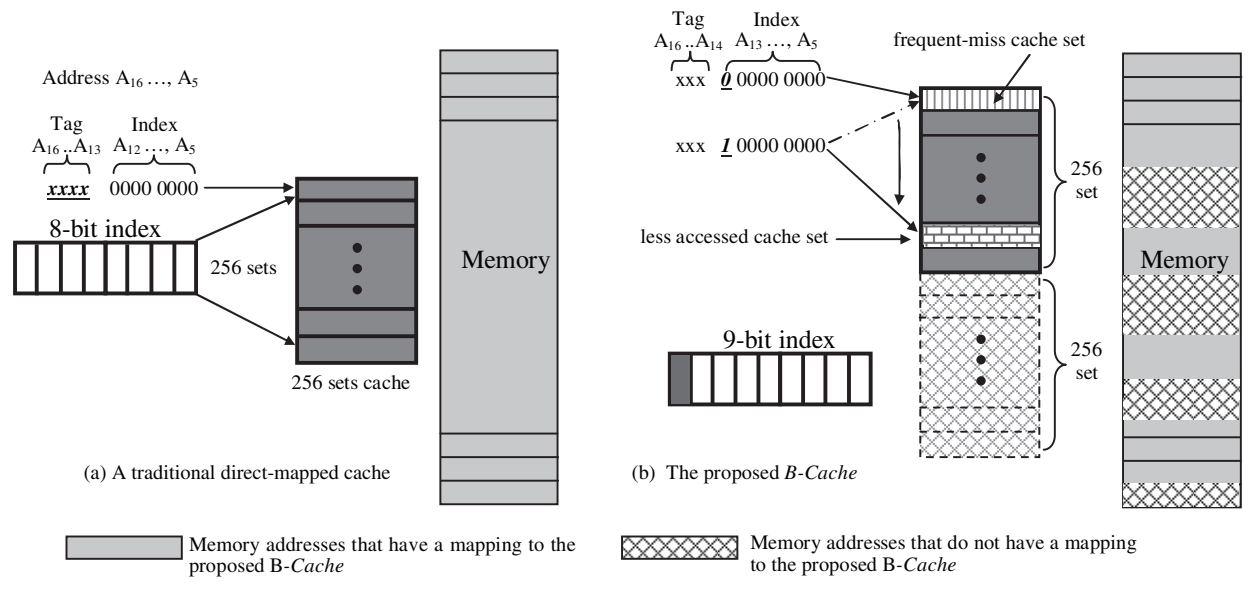

Fig. 4. A traditional direct-mapped cache and the proposed B-Cache.

accesses to each set, which means hardware overhead, and we need an extra cycle to locate these relocated cache sets [Peir et al. 1998], which causes performance overhead.

\subsection{Our Solution}

We solve the problem by balancing the accesses to the cache sets. From Figures 2 and 3, we observed that the cache misses occurred on frequent missed sets account for the major portion of the total cache misses. To reduce the cache misses, we should intuitively reduce the cache misses that occurred on these frequent missed sets. Figure 4 a shows a traditional direct-mapped cache where all memory addresses have a mapping to the cache set. Figure $4 \mathrm{~b}$ shows an example of the proposed $B$-Cache where the index is 1 bit longer than that of the same-sized direct-mapped cache. The misses occurring in frequent missed sets may be reduced by one-half, as shown in Figure 4b. In this example, we use a $4-\mathrm{kB}$ direct-mapped cache with a line size of 16 bytes in a 16-bit processor; the offset is 4 and the index is 8 bits (the address bits, cache size, and line size are chosen for easy illustration), the index is $A_{12} \ldots, A_{5}$, and the tag is $A_{16} \ldots$, $\mathrm{A}_{13}$. For the traditional direct-mapped cache, all memory addresses have a corresponding cache set determined by the 8-bit index. For the proposed B-Cache, we use a 9-bit index instead of an 8-bit index, but the total cache sets remain unchanged. Indexes of 9 bits require a maximum of 512 cache sets. Therefore, in this example, only one-half of the memory address has a mapping to the cache sets. Which memory address has a mapping is dynamically determined when there is a cache miss; thus, programmable decoders are used for the B-Cache.

The basic idea of the B-Cache is to decrease the accesses to the frequently missed cache sets and to increase the accesses to the underused cache sets. Figure 4b shows how the goal is achieved. The cache set with the index " 0000 0000 ," is a frequently missed cache set in the traditional direct-mapped cache. After increasing the index length from 8 bits to 9 bits, we can only map the address with an index of either "무,0000,0000" or " $\underline{\mathbf{1}}, 0000,0000$," to the original 


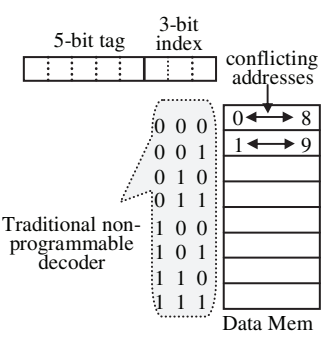

(a) Direct-mapped cache

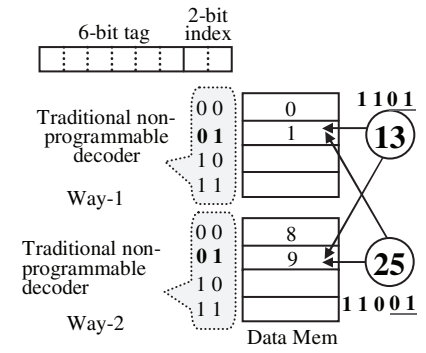

(b) 2-way cache

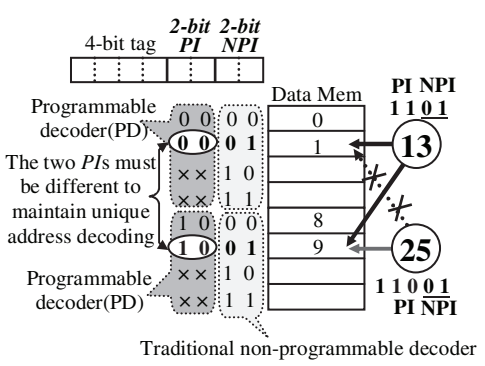

(c) Proposed B-Cache

Fig. 5. (a) A traditional direct-mapped cache; (b) a traditional 2-way cache; (c) the proposed $B$ Cache with programmable decoder. PI, programmable index; NPI: nonprogrammable index; " $\times$ " represents an invalid programmable decoder entry during an application's start-up.

set. Therefore, the proposed $B$-Cache has the potential to reduce the misses that occurred in this set by one half. In Figure $4 b$, the address with an index of "무, 0000,0000 " is mapped to the cache set. When the address with an index of " $1,0000,0000$ " is required by the processor, the cache controller has to find a victim cache set, which should be chosen from underutilized cache sets, so the cache space can be efficiently exploited. This is achieved by introducing a replacement policy, such as the LRU, into the B-Cache design. An underutilized cache set will become the least-recently used cache set, at last. We use a simple example to explain how the proposed $B$-Cache can balance the cache accesses and reduce the cache misses and how to program the programmable decoders during the cache block refill after a cache miss.

\subsection{Example}

Figure 5 shows a conventional direct-mapped cache (a), a conventional 2-way cache (b), and the proposed B-Cache (c). For simplicity, the cache contains only eight sets and the address contains only 8 bits. For an address sequence of 0,1 , $8,9, \ldots 0,1,8,9$, the direct-mapped cache experiences the worst results by having no cache hits at all. This occurs because the cache accesses are completely nonuniform, which is the case in real applications [Peir et al. 1998]. A traditional direct-mapped cache cannot adaptively accommodate these conflicting addresses, since the index decoding is fixed.

On the other hand, the 2-way cache exhibits cache hits after the first four warm-up accesses. The 2 -way cache achieves this high hit rate, since the decoder length is one bit less than the direct-mapped cache. During a miss, the 2-way cache has two candidates for the victim, while the direct-mapped cache has just one candidate. It is intuitive that decreasing the decoder length would generate more opportunities for choosing a better victim. The index lengths of 4 - and 8-way caches for this example are 1 and 0 bit, respectively.

Figure 5c shows the proposed B-Cache. We increase the decoder length by 1 bit. The first two most significant index bits are programmable indices for programmable decoders; the least two insignificant bits are nonprogrammable indices for nonprogrammable decoders. The outputs of the two decoders are 
ANDed together to control the activation of a word line. We will discuss how to determine the index length and how to divide the indices into programmable and nonprogrammable indices for a $B$-Cache in Section 6.

The $B$-Cache exhibits the same hit rate as the 2-way cache for this example. The defining feature of the B-Cache is the programmable decoder, which is programmed with the index of the desired address during a cache refill after a miss. For this simple example, there are two victim candidates, which are determined by the nonprogrammable index of an address. The programmable decoder will be dynamically programmed in the following three situations during a cache miss.

First, we consider the situation of a cache's cold start (start-up) of an application. In a multilevel memory hierarchy, miss ratio measurements are often made from a "cold start," that is, made with the higher-level cache initially empty [Easton and Fagin 1978]. This is the reason why there are valid bits for all the cache blocks in both instruction and data cache designs. The content of the programmable decoders are invalid. The contents of the programmable decoders are then programmed using the programmable index of each desired address. For addresses that have the same nonprogrammable index, such as addresses 0 and 8 , the victim is chosen using the replacement policy (least recently used replacement is assumed).

Second, we consider the situation in which the B-Cache exhibits a miss, but the programmable decoder exhibits a hit. For example, this occurs when address 25 is accessed after the aforementioned address sequence. The nonprogrammable index of the address 25 (11001) is " 01 " and the corresponding programmable index in the two programmable decoders are " 00 " and " 10 ," as shown in Figure 5c. Since the programmable index of the address 25 (11001) is " 10 ," the $B$-Cache exhibits a programmable decoder hit. Recall that since the $B$-Cache is a cache that only one cache block is activated during an access, address 25 must replace address 9 . In this situation, the $B$-Cache cannot choose a better victim based on the access history.

Last, both the $B$-Cache and the programmable decoder exhibit a miss. This occurs when address 13 is accessed after the aforementioned address sequence. The programmable index of address 13 (1101) is "11," which is different from the indices stored in the two programmable decoders, which are " 00 " and " $10 . "$ None of the programmable decoders are activated. It should be noted that this cache miss is not an extra miss caused by the limited address mapping. The same situation exists in fully associative caches using the whole tag as the decoding index and would not incur any extra misses. In fact, the programmable decoder predetermines the cache miss so neither data nor tag will be read out from the memory during a programmable decoder miss. Since the cache miss signal is, by default, true, this will not cause any problems. The victim for address 13 can be chosen from any of the two cache sets, based on the replacement policy.

Pointing out the difference between the B-Cache and the 2-way cache is important. For the 2 -way cache, both address " 13 " and " 25 " can be mapped to either of the two sets. Obviously, decreasing the programmable decoder hit rate during a cache miss would improve the $B$-Cache hit rate. A low programmable 


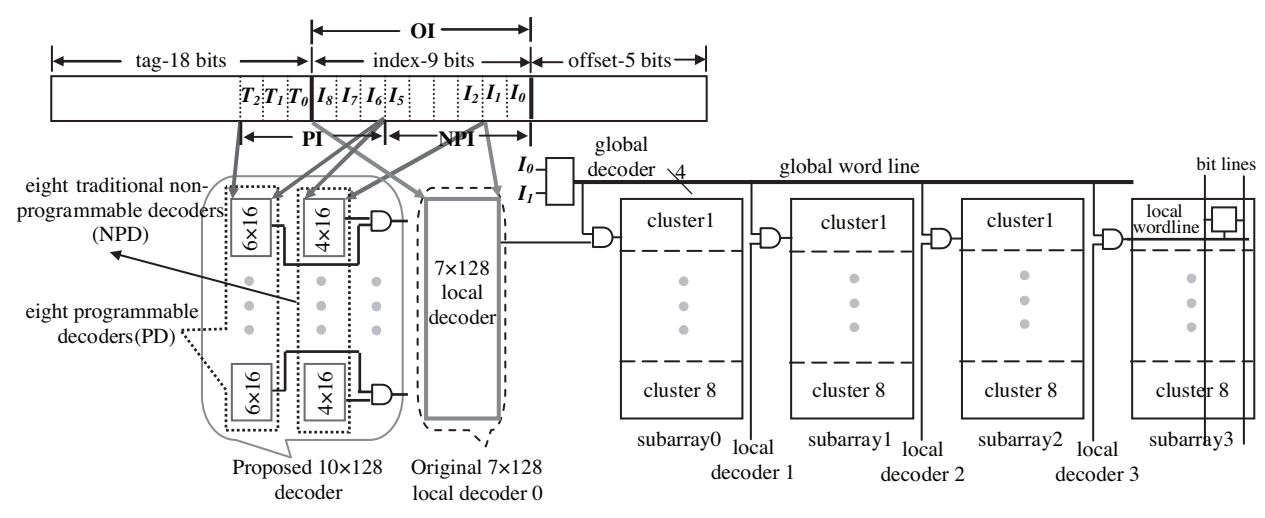

Fig. 6. B-Cache organization.

decoder hit rate means the replacement can be fully exploited to balance the cache accesses and reduce the cache misses.

\section{THE B-CACHE ORGANIZATION}

\subsection{Terminology}

The fundamental idea behind the $B$-Cache is to reduce the cache misses by balancing accesses to the cache sets. The defining property of the B-Cache is that the index length is longer than that in a conventional direct-mapped cache. We define the memory address mapping factor (MF)

$$
\mathrm{MF}=2^{(\mathrm{PI}+\mathrm{NPI})} / 2^{\mathrm{OI}}
$$

where $\mathrm{MF} \geq 1, \mathrm{PI}, \mathrm{NPI}$, and OI are the index lengths of the programmable decoder and nonprogrammable decoder of the $B$-Cache and the original directmapped cache, respectively. This means that $1 / \mathrm{MF}$ of the total memory addresses has a mapping to the cache sets after increasing the B-Cache's index length. We will show that larger values of MF decrease the programmable decoder hit rate during a cache miss in Section 4.3.2.

The $B$-Cache can reduce the miss rate, since multiple cache locations can be chosen for a victim, as shown in Figure 5c. We define the B-Cache associativity (BAS)

$$
\mathrm{BAS}=2^{\mathrm{OI}} / 2^{\mathrm{NPI}}
$$

where BAS $\geq 1$. This means the $B$-Cache logically divides the cache sets into BAS clusters, as shown in Figure 6 . For a cache miss, the victim can be chosen from these $B A S$ clusters. The miss rate of a $B$-Cache will approach the miss rate of a same-sized $B A S$-way cache.

The case $\mathrm{MF}=1$ or $\mathrm{BAS}=1$ is equivalent to a traditional direct-mapped cache. For B-Cache, both $\mathrm{MF}$ and BAS must be larger than one to achieve a lower miss rate than traditional direct-mapped caches. 


\subsection{Organization}

Cache memory is partitioned into a number of identically sized subarrays to tradeoff the access time, area, and power consumption [Ghose and Kamble 1999; Reinmann and Jouppi 1999]. Each subarray stores a part of the accessed word or several words, depending on the divisions of cache memory from both the word line and the bit line cuts.

Figure 6 shows the organization of a 16-kB direct-mapped cache with a line size of 32 bytes. The address is assumed to have $32 \mathrm{bits}$. The data memory is partitioned into four subarrays and the tag memory is partitioned into eight subarrays (not shown in the figure) [Reinmann and Jouppi 1999]. Each subarray has its own local decoder. The divided word-line technique [Yoshimoto et al. 1983 ] is adopted to achieve both the fast access time and low per access energy consumption. For the ease of illustration, we use the least significant 2 bits of the index, $I_{1}$ and $I_{0}$ as the inputs to the global word-line decoder to determine the selection of a subarray. The other 7 bits, from $I_{8}$ to $I_{2}$, determine the cache line selection in a particular subarray. The combination of the global and local word line locates a particular set for an address.

It should be noted that any address bit combination can be used as the global and local word-line decoder inputs and the B-Cache is not restricted to any index-decoding scheme or cache memory partitions. There does exist optimization opportunities in selecting the address bits for index decoding to achieve a low miss rate; however, indexing optimization [Givargis 2003] is out of the range of this paper.

For the $B$-Cache, we replace the original $7 \times 128$ local decoders with new $10 \times 128$ decoders. The new decoder includes eight $4 \times 16$ nonprogrammable decoders and eight $6 \times 16$ programmable decoders. The nonprogrammable decoder uses index bits $\mathrm{I}_{5}$ to $\mathrm{I}_{2}$, while the programmable decoder uses $\mathrm{I}_{8}, \mathrm{I}_{7}, \mathrm{I}_{6}$, and three tag bits $\mathrm{T}_{2}, \mathrm{~T}_{1}$, and $\mathrm{T}_{0}$ as inputs. For this particular design, the $B$-Cache's two parameters are $\mathrm{MF}=8$ and $\mathrm{BAS}=8$. We determine the $\mathrm{MF}$ and BAS through experimentation and, thus, defer the discussion of how to choose $B$-Cache parameters in Sections 4.3.1 and 4.3.2.

\subsection{The B-Cache's Replacement Policy}

A $B$-Cache increases the accesses to underutilized sets, balances the cache access, and reduces the cache misses through the replacement policy. Any replacement policy available to conventional set-associative caches is applicable to the $B$-Cache. We evaluate both random replacement and the LRU policy for the $B$-Cache design. The random policy is simple to design and needs negligible extra hardware. The LRU may achieve a better hit rate, but will have more area overhead than the random policy. Designing a new replacement policy specifically for a $B$-Cache to detect the underutilized sets and, hence, choose the victim accordingly, may further reduce the miss rate. However, we show in Section 4.3.1 that the $B$-Cache's miss rate reduction approaches an 8-way cache, which makes further reducing the miss rate through innovative replacement policies unimportant. 


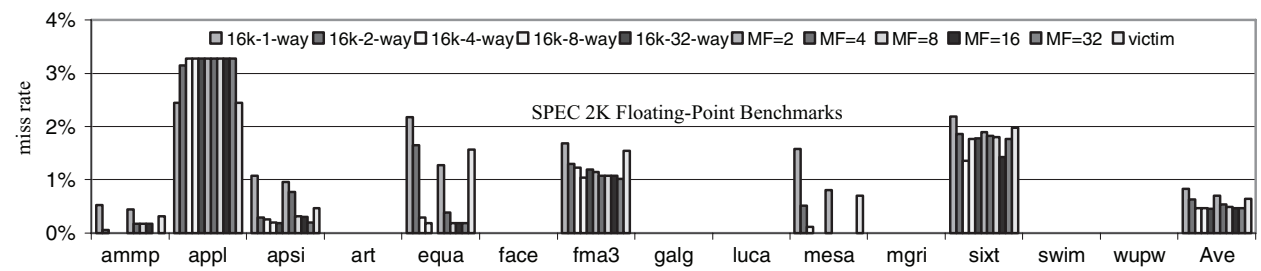

Fig. 7. Instruction cache miss rates of a direct-mapped, 2-, 4-, 8-, and 32-, the $B$-Cache with $\mathrm{MF}=$ $2,4,8,16$, or 32 and $\mathrm{BAS}=8$ and 16-entry victim buffer with a size of $16 \mathrm{kB}$ (replacement policy is LRU).

\section{EXPERIMENTAL METHODOLOGY AND RESULTS}

We use miss rate as the primary metric to measure the $B$-Cache effectiveness. We used an eight-issue out-of-order processor simulator to collect the miss rate. We determine the $B$-Cache parameters MP and BAS through experimentation. Overall performance improvement and energy is discussed in Section 6.1.

\subsection{Cache Hierarchy}

The baseline $\mathrm{L} 1$ cache is a $16-\mathrm{kB}$ direct-mapped cache with a line size of 32 bytes for both the instruction and data caches. We use a unified 4-way $256-\mathrm{kB} \mathrm{L-2}$ cache, using LRU replacement policy and a hit latency of six cycles. The results of $\mathrm{L} 1$ cache sizes of 8 and $32 \mathrm{kB}$ are also collected to show the effectiveness of the B-Cache.

\subsection{Benchmarks}

We run all 26 SPEC2K benchmarks using the SimpleScalar tool set [Burger and Austin 1997]. The benchmarks are precompiled for the Alpha ISA and obtained from the SimpleScalar developers [Weaver]. In the original version of this paper [Zhang 2006], the benchmarks were fast-forwarded for 2 billion instructions and executed for 500 million instructions afterward, using reference inputs. However, reports indicate that these arbitrarily selected simulation points may not reveal the real performance behavior of the benchmarks. Therefore, we use SimPoint [Sherwood et al. 2002] to choose the simulation points in our new experiments and each benchmark is executed for 100-M instructions. In the original paper, the instruction cache results of some benchmarks, whose miss rates are less than $0.01 \%$, were not reported to save space in the plot. These benchmarks included applu, art, bzip, facerec, galgel, gzip, lucas, mcf, mgrid, swim, and vpr. All results, however, are reported in this paper.

\subsection{Experimental Results}

The miss rates (instead of miss rate reductions over the baseline, as in the original paper) of eleven different cache configurations, including the baseline, are shown in Figures 7, 8, 9, and 10. The results are reported in groups of CINT2K (integer) and CFP2K (floating-point) components of SPEC2K. Four traditional set-associative cache configurations (2-, 4-, 8-, and 32-way) are included to show the miss rate reductions over the baseline. The miss rate of a 16-entry victim 


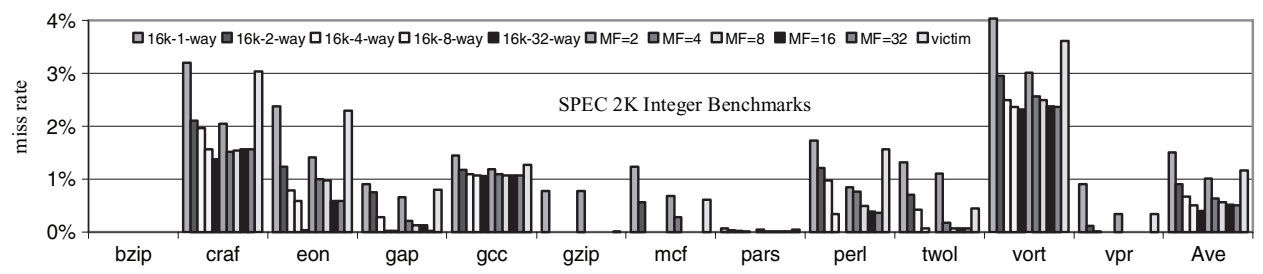

Fig. 8. Instruction cache miss rates of a direct-mapped, 2-, 4-, 8-, and 32-way, the B-Cache with $\mathrm{MF}=2,4,8,16$, or 32 and $\mathrm{BAS}=8$, and 16-entry victim buffer with a size of $16 \mathrm{kB}$ (replacement policy is LRU).

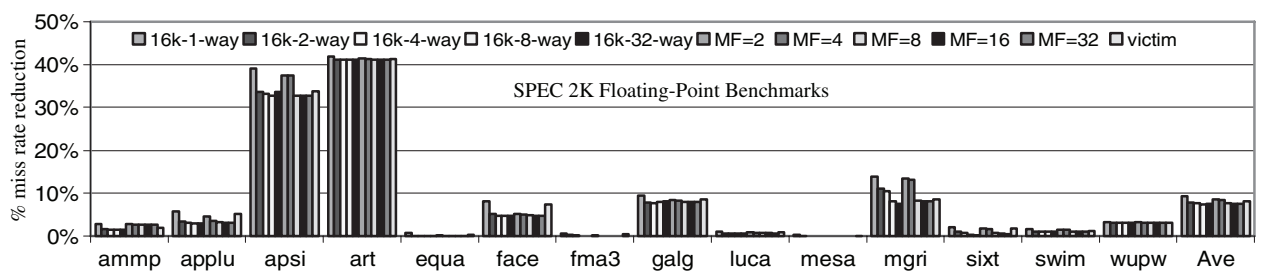

Fig. 9. Data cache miss rates of a direct-mapped, 2-, 4-, 8-, and 32-way, the $B$-Cache with $\mathrm{MF}=2$, $4,8,16$, or 32 and $\mathrm{BAS}=8$, and 16-entry victim buffer with a size of $16 \mathrm{kB}$ (replacement policy is LRU).

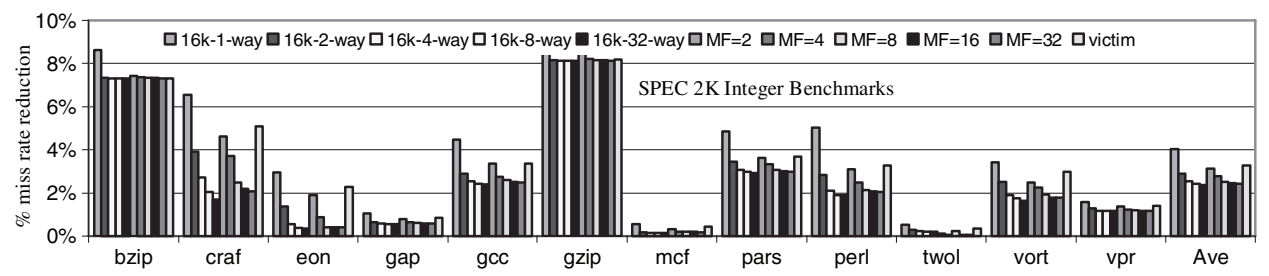

Fig. 10. Data cache miss rates of a direct-mapped, 2-, 4-, 8-, and 32-way, the B-Cache with $\mathrm{MF}=$ $2,4,8,16$, or 32 and $\mathrm{BAS}=8$, and 16-entry victim buffer with a size of $16 \mathrm{kB}$ (replacement policy is LRU).

buffer is included and will be compared with the B-Cache design in Section 6.6. The other five configurations include the $B$-Cache at the memory mapping factor $\mathrm{MF}=2,4,8,16$, or 32 with a $B$-Cache associativity of $\mathrm{BAS}=8$. The bar marked Ave was computed as the average miss rate for all of the benchmarks.

4.3.1 The B-Cache Associativity-BAS. We determine the B-Cache associativity, BAS, through experimentation. From Figures 7, 8, 9, and 10, we can see that increasing associativity higher than eight would not bring significant miss rate reductions. On average, the miss rate of the 32 -way cache is only $0.1 \%$ lower than the 8 -way cache for an instruction cache on integer benchmarks. We observe a meaningful miss rate reduction for benchmarks, crafty, eon, and perlmk, where they show a $0.2,0.5$, and $0.3 \%$ miss rate reduction over the 8-way cache. For data cache, the average miss rate of the 32 -way cache is $0.03 \%$ lower than the 8 -way cache. On the other hand, the 8 -way cache outperforms the 4 -way cache by 0.03 and $0.3 \%$ on CFP2K for the instruction and data 


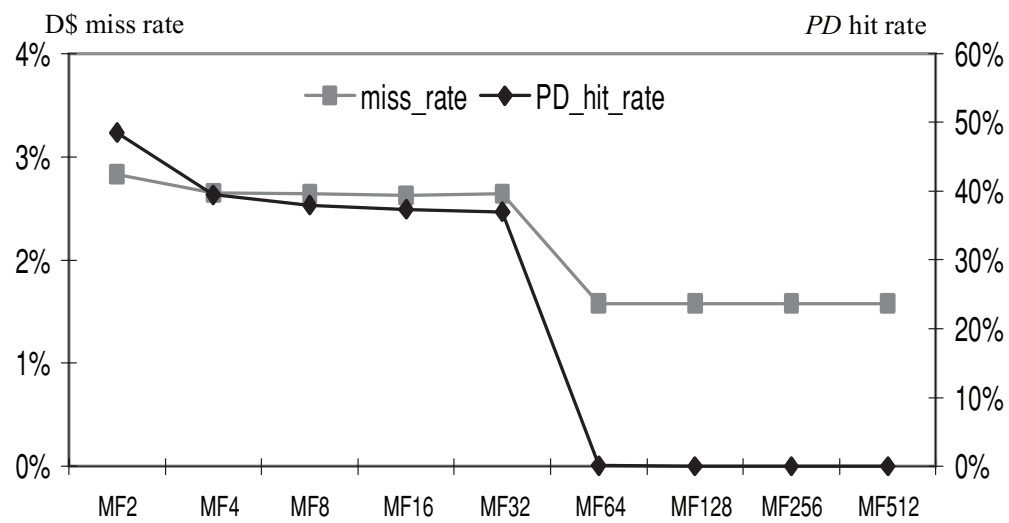

Fig. 11. Data cache miss rate (left) and programmable decoder (PD) hit rate (right) of the benchmark ammp at cache size of $16 \mathrm{kB}$ with varied MP values.

cache, respectively. For some benchmarks, crafty and perlbmk, the 8-way cache has a 0.4 and $0.6 \%$ lower miss rate than a 4 -way instruction cache; the same situation exists in the data cache for benchmarks crafty and mgrid. Since the miss rate of a $B$-Cache with associativity $\mathrm{BAS}=8$ would approach the miss rate of an 8-way cache, we choose BAS $=8$. Further increasing the BAS to 16 or higher would not reduce the miss rate significantly, but may incur more overhead in programmable decoders, which is discussed in Section 5.

It should be noted that all the miss rates are reported as the real value of miss rates instead of the relative miss rate reductions over the baseline, as we did in the original paper. The benefit of reporting the real miss rate is that some benchmarks already have a very low miss rate and the relative miss rate reduction may not be important to performance improvements. We will further show the difference of reporting real values and the relative reduction on IPCs in Section 6.1.

4.3.2 Memory Address Mapping Factor-MF. We determine the optimal MF through experimentation. Figures 7, 8, 9, and 10, also show the miss rates when the MF of the proposed $B$-Cache is increased from 2, 4, 8, 16, to 32. The miss rates of the $B$-Cache for both the data and instruction caches on all 26 SPEC2K benchmarks are reduced significantly when the MF is increased from two to four, and then continue to be increased to eight. Further increasing the MF to 16 or 32 improves the miss rate of instruction cache by a mere 0.05 and $0.02 \%$ and the data cache by a mere of 0.04 and $0.07 \%$ for CINT2K and CFP2K benchmarks, respectively.

There are some benchmarks, such as ammp and galgel, whose data miss rates reductions of the $B$-Cache are lower than the 4-way cache. We increase the MF and measure the $B$-Cache's miss rate and programmable decoder hit rate during a cache miss for the benchmark, ammp. The results are shown in Figure 11. The miss rate decreases simultaneously with the decoder hit rate do. This is because during a programmable decoder hit, the B-Cache cannot fully exploit the replacement policy. When the MF is increased from 32 to 64, 


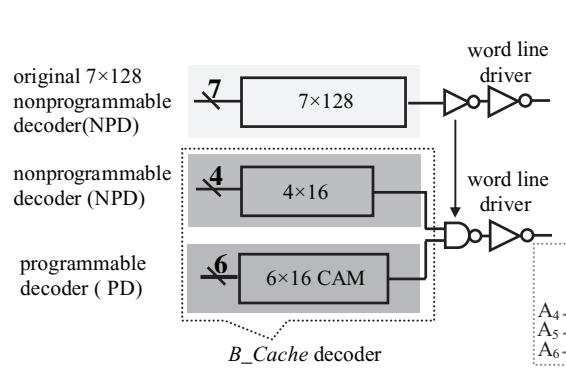

(a) The original $7 \times 128$ and the $B$-Cache's $4 \times 16$ nonprogrammable decoder

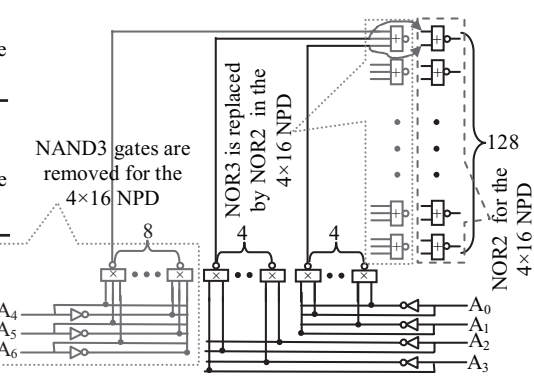

(b) The circuits of the original $7 \times 128$ and the $B$-Cache's eight $4 \times 16 \mathrm{NPD}$

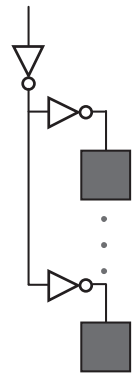

(c) search bitline

Fig. 12. Decoder timing analysis.

the programmable decoder hit rate is reduced sharply and so is the cache miss rate. However, a larger MF means more bits for the programmable decoder and, thus, more overhead in terms of area and access time. Considering the access time analysis of the $B$-Cache in Section 5.1, we choose a memory address mapping factor $\mathrm{MF}=8$.

4.3.3 Miss Rate Reduction of the B-Cache. The upper boundary on the miss rate reduction of the $B$-Cache with BAS $=8$ is the miss rate of an 8-way cache. It approaches the upper boundary as the memory address-mapping factor, MF, increases. From Figures 7, 8, 9, and 10, the miss rate reduction of the B-Cache is as good as a 4-way cache for both the data and instruction caches.

\section{PROGRAMMABLE DECODER DESIGN}

In this section, we evaluate the latency, storage, and power costs associated with the $B$-Cache. Storage is measured in terms of equivalent SRAM bits. Cacti v4.2 is used to model the access time and energy of both the B-Cache and the traditional direct-mapped caches.

\subsection{Timing Analysis}

The critical path of a conventional direct-mapped cache normally resides on the tag side instead of the data side [Peir et al. 1996]. The B-Cache incorporates three tag bits into the programmable decoder; therefore, the tag length is reduced by 3 bits, which reduces the tag-side access time. However, we do not claim this access time reduction, since the data and tag path may already be balanced [Peir et al. 1996], so that the data side would be on the critical path.

The $B$-Cache modifies the local decoder without changing the global decoders. Therefore, the B-Cache's local decoder should run faster than, or at least as fast as, that of the original local decoder of each subarray to avoid increasing the access time. The $B$-Cache decoder consists of a programmable and nonprogrammable decoder, as shown in Figure 12a. The original $7 \times 128$ decoder is composed of two 2-input NAND gates and one 3-input NAND gate, as shown in Figure 12b. The outputs of these NAND gates are ORed through a 3-input NOR gate. The nonprogrammable decoder in the B-Cache decoder has two 
Table II. Timing Analysis of the Decoders for Traditional and B-Cache ${ }^{a}$

\begin{tabular}{|c|c|c|c|c|c|c|c|c|c|c|}
\hline \multirow{2}{*}{$\begin{array}{l}\text { Traditional } \\
\text { Decoder }\end{array}$} & \multicolumn{2}{|c|}{$8 \times 256$} & \multicolumn{2}{|c|}{$7 \times 128$} & \multicolumn{2}{|c|}{$6 \times 64$} & \multicolumn{2}{|c|}{$5 \times 32$} & \multicolumn{2}{|c|}{$4 \times 16$} \\
\hline & \multicolumn{2}{|c|}{ 3D_3R } & \multicolumn{2}{|c|}{ 3D_3R } & \multicolumn{2}{|c|}{ 2D_3R } & \multicolumn{2}{|c|}{ 3D_2R } & \multicolumn{2}{|c|}{ 2D_2R } \\
\hline \multicolumn{11}{|l|}{ Time (ns) } \\
\hline $0.18 \mu \mathrm{m}$ & \multicolumn{2}{|c|}{0.554} & \multicolumn{2}{|c|}{0.476} & \multicolumn{2}{|c|}{0.315} & \multicolumn{2}{|c|}{0.269} & \multicolumn{2}{|c|}{0.234} \\
\hline $0.13 \mu \mathrm{m}$ & \multicolumn{2}{|c|}{0.431} & \multicolumn{2}{|c|}{0.370} & \multirow{2}{*}{\multicolumn{2}{|c|}{0.235}} & \multicolumn{2}{|c|}{0.196} & \multicolumn{2}{|c|}{0.170} \\
\hline $0.09 \mu \mathrm{m}$ & \multicolumn{2}{|c|}{0.336} & \multicolumn{2}{|c|}{0.286} & 0.171 & & \multicolumn{2}{|c|}{0.138} & \multicolumn{2}{|c|}{0.119} \\
\hline \multirow{2}{*}{ BC decoder } & PD & NPD & PD & NPD & $\mathrm{PD}$ & NPD & $\mathrm{PD}$ & NPD & PD & NPD \\
\hline & $6 \times 32$ & $5 \times 32$ & $6 \times 16$ & $4 \times 16$ & $6 \times 8$ & $3 \times 8$ & $6 \times 4$ & $2 \times 4$ & $6 \times 2$ & $1 \times 2$ \\
\hline Composition & CAM & 3D_2R & CAM & 2D_2R & CAM & NAND3 & CAM & NAND2 & CAM & INV \\
\hline \multicolumn{11}{|l|}{ Time (ns) } \\
\hline $0.18 \mu \mathrm{m}$ & 0.493 & 0.534 & 0.437 & 0.446 & 0.294 & 0.301 & 0.259 & 0.256 & 0.228 & 0.223 \\
\hline $0.13 \mu \mathrm{m}$ & 0.361 & 0.415 & 0.317 & 0.354 & 0.215 & 0.218 & 0.188 & 0.182 & 0.159 & 0.157 \\
\hline $0.09 \mu \mathrm{m}$ & 0.254 & 0.312 & 0.222 & 0.265 & 0.148 & 0.158 & 0.134 & 0.127 & 0.107 & 0.102 \\
\hline
\end{tabular}

${ }^{a}$ PD, programmable decoder; NPD, nonprogrammable decoder. The composition of the decoders are represented in NAND and NOR gates; 2D_3R stands for 2-input NAND gate and 3-input NOR gate.

2 -input NAND gates. The outputs of the 2-input NAND gates are ORed through a 2-input NOR gate.

The programmable decoder of the B-Cache is composed of eight $6 \times 16$ CAMs. We need an AND gate for the outputs of the nonprogrammable and programmable decoder of the $B$-Cache. Inserting a new AND gate would increase the access time. The technique proposed in Zhang et al. [2003] is employed, which changes the inverter in the original word line driver to a 2-input NAND gate. The transistor sizes of the 2 -input NAND are selected to make the NAND gate as fast as the original inverter and, thus, incurs no access time overhead. By using this technique, if there is time remaining left between the $B$-Cache and the original decoder, the B-Cache would not prolong the B-Cache's access time.

Table II shows the access time for the decoders of $8 \times 256,7 \times 128,6 \times 64$, $5 \times 32$, and $4 \times 16$, which corresponds to the subarray sizes of $8,4,2$, and $1 \mathrm{kB}$, and 512 bytes with a cache line size of 32 bytes. To date, we have not found any $\mathrm{L} 1$ cache memory whose subarray size is larger than $8 \mathrm{kB}$ or less than 512 bytes [Reinmann and Jouppi 1999]. For each original decoder, the corresponding $B$-Cache decoder is shown. The access time of both the conventional and the programmable decoders for the $B$-Cache are calculated from the Cacti 4.2, at technologies $0.18,0.13$, and $0.09 \mu \mathrm{m}$. From this table, we can see that all of the decoders have time remaining; therefore, the $B$-Cache would not have any time access overhead compared with a conventional direct-mapped cache.

One may ask how the B-Cache's decoder is as fast as the original directmapped cache, given that the index length of the B-Cache is 3 bits longer than the original index. Compared with the baseline, the $B$-Cache's $4 \times 16$ decoder is faster than the original $7 \times 128$ decoder, since the NOR2 gates, which are faster, replace the NOR3 gates. The critical path of the decoder, for this example, is also changed from the 3 - to the 2 -input NAND gates. However, we should point out that the $B$-Cache's $4 \times 16$ nonprogrammable decoder is much slower than the $4 \times 16$ decoder in the original direct-mapped cache with a subarray size of 


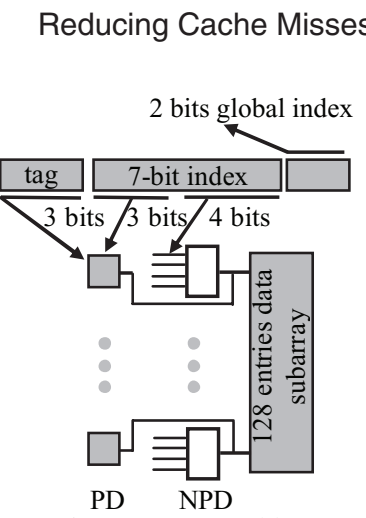

(a) data memory partition

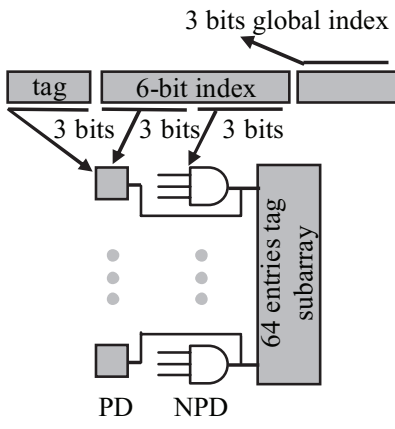

(b) tag memory partition

Fig. 13. Programmable decoder design when memory partition in tag and data is different. PD, programmable decoder, NPD: nonprogrammable decoder. The 4-input AND gates are implemented through two stages of NAND and NOR gates.

512 bytes, as shown in Table II This is because they have different subarray dimensions and output loads. The 2-input NAND gate in the B-Cache's $4 \times 16$ decoder has a fan out of $8 \times 4=32$ gates, while the fan out of the $4 \times 16$ in the conventional direct-mapped cache is four gates. This explains why the $7 \times 128$ decoder in the conventional $16 \mathrm{kB}$ direct-mapped cache has not been divided into one $4 \times 128$ and one $3 \times 128$ and then combined through the NAND gate (changed from the inverter). The performance improvement is less than $0.5 \%$ with an area overhead of 10242 -input NOR gates and enlarged inverters.

To speed up the CAM comparison, the search bit lines of the CAM are segmented, as shown in Figure 12c, which is similar to the technique proposed in [Ghose and Kamble 1999]. One search bit line needs nine extra inverters. For this $16-\mathrm{kB}$ direct-mapped cache, $(6 \times 9+2 \times 6 \times 9) \times 4=648$ inverters are required, which represents a fraction of the total area.

\subsection{Different Partitions of Tag and Data Memory}

Tag and data memory typically use different memory partitions to achieve the best tradeoff across performance, power, and area. For example, the data memory is partitioned into four subarrays, while tag memory is partitioned into eight subarrays for this $16-\mathrm{kB}$ direct-mapped cache. Figure 13 shows the decoder design for tag and data memory subarrays for the B-Cache. For the tag decoder, eight subarrays need three bits for the global decoder, while the global data decoder requires two bits for four subarrays. Since both tag and data decoders must have the same $B$-Cache parameters $(\mathrm{MF}=8, \mathrm{BAS}=8$ ) and programmable index length $\mathrm{PI}=6$, the length of the nonprogrammable decoder for the $B$-Cache is 4 and 3 bits for the data and tag decoder, respectively. From Table II, we can see the decoders' access times are faster than the original design.

\subsection{Subarray Width and Cache Line Width}

The design of the $B$-Cache requires that the subarray width is the same as or less than the cache line width, since the tag is embedded into the decoder for each cache block. There are six parameters for a cache memory organization. 
Table III. Subarray Width and Cache Block Width at Cache Sizes of 8, 16, 32, and $64 \mathrm{kB}$ at Line Sizes of 32 and 64 Bytes at Technologies of $0.18,0.13 \mu \mathrm{m}$, and $0.09 \mu \mathrm{m}$

\begin{tabular}{|c|c|c|c|c|c|c|c|c|c|c|c|c|}
\hline Decoder & \multicolumn{3}{|c|}{$8 \mathrm{kB}$} & \multicolumn{3}{|c|}{$16 \mathrm{kB}$} & \multicolumn{3}{|c|}{$32 \mathrm{kB}$} & \multicolumn{3}{|c|}{$64 \mathrm{kB}$} \\
\hline Composition & \multicolumn{3}{|c|}{ 3D_3R } & \multicolumn{3}{|c|}{ 2D_3R } & \multicolumn{3}{|c|}{ 3D_2R } & \multicolumn{3}{|c|}{ 2D_2R } \\
\hline Linesize $=32 B$ & Ndwl & Ndbl & Nspd & $\mathrm{Ndwl}$ & Ndbl & Nspd & $\mathrm{Ndwl}$ & Ndbl & Nspd & Ndwl & Ndbl & Nspd \\
\hline \multicolumn{13}{|l|}{ Data array } \\
\hline $0.18 \mu \mathrm{m}$ & 1 & 8 & 1 & 1 & 8 & 1 & 1 & 16 & 1 & 1 & 32 & 1 \\
\hline $0.13 \mu \mathrm{m}$ & 1 & 8 & 1 & 1 & 8 & 1 & 1 & 16 & 1 & 1 & 32 & 1 \\
\hline $0.09 \mu \mathrm{m}$ & 1 & 8 & 1 & 1 & 16 & 1 & 1 & 16 & 1 & 1 & 32 & 1 \\
\hline Linesize $=32 B$ & Ntwl & Ntbl & Nspt & Ntwl & Ntbl & Nspt & Ntwl & Ntbl & Nspt & Ntwl & Ntbl & Nspt \\
\hline \multicolumn{13}{|l|}{ Tag array } \\
\hline $0.18 \mu \mathrm{m}$ & $\underline{1}$ & $\underline{8}$ & $\underline{2}$ & $\underline{1}$ & $\underline{32}$ & $\underline{2}$ & 4 & 32 & 4 & 4 & 32 & 4 \\
\hline $0.13 \mu \mathrm{m}$ & 1 & 16 & 1 & 1 & $\overline{32}$ & 1 & 4 & 32 & 4 & 4 & 32 & 4 \\
\hline $0.09 \mu \mathrm{m}$ & 1 & 32 & 1 & 32 & 4 & 16 & 32 & 4 & 32 & 4 & 32 & 4 \\
\hline Linesize $=64 B$ & Ndwl & Ndbl & Nspd & Ndwl & Ndbl & Nspd & $\mathrm{Ndwl}$ & Ndbl & Nspd & Ndwl & $\mathrm{Ndbl}$ & Nspd \\
\hline \multicolumn{13}{|l|}{ Data array } \\
\hline $0.18 \mu \mathrm{m}$ & 1 & 8 & 0.5 & 1 & 8 & 1 & 1 & 8 & 1 & 1 & 16 & 1 \\
\hline $0.13 \mu \mathrm{m}$ & 1 & 8 & 0.5 & 1 & 8 & 1 & 1 & 8 & 1 & 1 & 32 & 0.5 \\
\hline $0.09 \mu \mathrm{m}$ & 1 & 8 & 0.5 & 1 & 16 & 0.5 & 1 & 8 & 1 & 1 & 32 & 0.5 \\
\hline Linesize $=64 B$ & Ntwl & Ntbl & Nspt & Ntwl & Ntbl & Nspt & Ntwl & Ntbl & Nspt & Ntwl & Ntbl & Nspt \\
\hline \multicolumn{13}{|l|}{ Tag array } \\
\hline $0.18 \mu \mathrm{m}$ & $\underline{1}$ & $\underline{8}$ & $\underline{2}$ & 1 & 32 & 1 & 4 & 32 & 2 & 4 & 32 & 4 \\
\hline $0.13 \mu \mathrm{m}$ & 1 & $\underline{8}$ & 2 & 1 & 32 & 1 & 1 & 32 & 1 & 4 & 32 & 4 \\
\hline $0.09 \mu \mathrm{m}$ & 32 & 1 & 16 & 32 & 1 & 32 & 32 & 4 & 16 & 32 & 4 & 32 \\
\hline
\end{tabular}

Two parameters, Ndwl and Ndbl, indicate the divisions of the word and bitlines of the data memory array. Nspd indicates how many sets are mapped to a single wordline of a data memory. The other three parameters, Ntwl, Ntbl, and Nspt, represent the division of the tag memory. Table III shows these six parameters of caches at sizes of $8,16,32$, and $64 \mathrm{kB}$, with cache line sizes of 32 and 64 bytes at technologies of $0.18,0.13$, and $0.09 \mu \mathrm{m}$. There are a total of 24 different cache configurations; four of the cache memory array's Nspd are larger than Nwdl, which means there is more than one set in one wordline.

In these four situations, there are three cases at cache sizes of $8 \mathrm{kB}$, at technology $0.18 \mu \mathrm{m}$ with line sizes of both 32 and 64 bytes and at the technology of $0.13 \mu \mathrm{m}$ at a line size 64 bytes. The fourth situation is a cache size of $16 \mathrm{kB}$ at a line size of 32 bytes at the technology $0.18 \mu \mathrm{m}$. To meet the requirement of the $B$-Cache, we increase the word line division and make the parameter of Ntwl equal to Nspd. Since the tag memory is further divided, the access time is not increased, but decreased by $0.1 \%$, with an area increased by $0.5 \%$ and power per access increased by $1 \%$. In conclusion, the overhead of meeting the $B$-Cache requirement is trivial.

\subsection{Storage Overhead}

The additional hardware for the B-Cache is the CAM-based programmable decoder. The area [Efthymio and Garside 2002] of the CAM cell is 25\% larger than the SRAM cell used by data and tag memory. There are sixty-four $6 \times 8$ and thirty-two $6 \times 16$ CAMs. The total storage requirements for both the baseline and the B-Cache are calculated in Table IV. The overhead of the B-Cache 
Table IV. Storage Cost Analysis

\begin{tabular}{|c|c|c|c|c|c|}
\hline & Tag Dec & Tag Mem & Data Dec & Data Mem & Total size \\
\hline Baseline & No Mem cell & 20 bit $\times 512$ & No Mem cell & 256 bit $\times 512$ & 141,312 \\
\hline$B$-Cache & $64 \times 6 \times 8$ CAM & 17 bit $\times 512$ & $32 \times 6 \times 16$ CAM & 256 bit $\times 512$ & 147,456 \\
\hline
\end{tabular}

Table V. Energy per Cache Access ${ }^{a}$

\begin{tabular}{|c|c|c|c|c|c|c|c|c|}
\hline & T_SA & T_Dec & T_BL_WL & D_SA & D_Dec & D_BL_WL & D_others & Total (pJ) \\
\hline Baseline & 74.5 & 16.2 & 20.7 & 144.4 & 66.8 & 118.1 & 32.7 & 473.4 \\
\hline B_Cache & 63.3 & $8.4+64 \times 0.78$ & 17.6 & 144.4 & $36.9+32 \times 1.62$ & 118.1 & 32.7 & 523.1 \\
\hline
\end{tabular}

${ }^{a} \mathrm{~T}$, tag; D, data; SA, sense amplifier; Dec, decoder; BL, bitline; WL, word line.

increases the total cache area of the baseline by $4.3 \%$, which is less than a same-sized 4-way cache without considering the area used for implementing the replacement policy.

\subsection{Power Overhead}

The extra power consumption comes from the programmable decoder of each subarray. Data and tag memory have four and eight subarrays, respectively. We compute the power consumption of the programmable decoder using Cacti 4.2 at $0.18 \mu \mathrm{m}$ technology. A $6 \times 8$ and $6 \times 16$ CAM decoder consumes 0.78 and $1.62 \mathrm{pJ}$ per search, respectively. The B-Cache uses sixty-four $6 \times 8$ CAMs for tag-programmable decoders and thirty-two $6 \times 16$ CAMs for dataprogrammable decoders. We calculate the power reductions resulting from the 3 -bit tag length reduction and the removal of the 3-input NAND gates in the original tag and data decoders using Cacti 4.2. The energy per cache access of both the baseline and the B-Cache are shown in Table V. The power consumption of the $B$-Cache is $10.5 \%$ higher than the baseline; however, it is still 17.4 , 44.4 , and $65.5 \%$ lower than the same-sized 2-, 4-, and 8-way caches, respectively. Described in Section 6.2, the overall energy analysis of the B-Cache shows that the $B$-Cache reduces the overall memory-related energy consumption by $12 \%$ over the baseline because of the reduced miss rate and, hence, the reduction to the application's execution time.

\section{ANALYSIS}

In this section, we present the impact of the $B$-Cache on overall processor performance and energy consumption. We also discuss the tradeoffs of the $B$-Cache design parameters. We evaluate the balance of the $B$-Cache and compare it to other related techniques. Finally, we discuss the issues involving virtually or physically addressed tagged caches.

\subsection{Overall Performance}

Table VI shows the processor configuration for both the baseline and the proposed B-cache. Figures 14 and 15 show the IPC of the processor when the cache is configured as direct-mapped, 2-, 4-, 8-, and 32-way, the proposed B-Cache at $\mathrm{MF}=2,4,8,16$, or 32 , and a victim buffer with 16 lines. 
Table VI. Baseline and the B-Cache Processor Configuration

\begin{tabular}{|l|l|}
\hline Fetch/issue/retire width & \multicolumn{1}{|c|}{8 Instructions/cycle, 8 functional units } \\
\hline Instruction window size & 16 Instructions \\
\hline L1 cache & $16 \mathrm{kB}, 32 \mathrm{~B}$ linesize, direct-mapped \\
\hline L2 unified cache & $128 \mathrm{kB}, 128 \mathrm{~B}$ linesize, 4 -way, 6 cycle hit \\
\hline Main memory & Infinite size, 150 cycle access \\
\hline
\end{tabular}

Figures 16 and 17 show the performance improvements measured in the IPC of the processor with the above cache configuration over the baseline processor. We observe significantly different results depending on whether the real values of the IPCs or the relative improvements of IPCs over the base line are evaluated.

The results are reported in the groups of the integer and floating-point benchmarks, since they reveal totally different behaviors. The bar of Ave is computed as the arithmetic average of the benchmarks in a figure. When using the real value of the IPCs, the performance of the processor with the $B$-Cache outperforms the baseline by an average of 21.5 and $42.3 \%$ over the baseline for the integer and floating-point benchmarks, respectively. The greatest performance improvement is seen in equake, where the IPC increases from 1.3 to 3.0. The performance of the B-Cache is only 0.015 less than the 8 -way cache, but is 0.24 higher than the victim buffer.

When using the relative IPC improvements over the baseline, as shown in Figures 14 and 15, we see 52.9 and $36.7 \%$ IPC improvement for integer and floating-point benchmarks, respectively. The highest IPC improvement is seen in the benchmark, apsi. The IPC is increased by $430 \%$ over the baseline. Therefore, the average IPC improvement (results from Figure 16) using relative value is much higher than the improvement of average IPC (results from Figure 14) for the integer benchmarks. On the other hand, for floating-point benchmarks, the average IPC improvement (results from Figure 15) is lower than the improvements of average IPC (results from Figure 17).

\subsection{Overall Energy}

There are two main components that result in power dissipation in CMOS circuits, namely, static power dissipation because of the leakage of current and dynamic power dissipation as a result of logic switching current and the charging and discharging of the load capacitance. We consider both types of energy in our evaluation. We evaluate the memory-related energy consumption [Zhang et al. 2003] including on chip caches, as described in Section 4.1, and off-chip memory. Figure 18 lists the equations for computing the total memory-related energy consumption.

The terms in italic are those obtained through measurements or simulations. We compute cache_access, cache_miss, and cycles by running SimpleScalar simulations for each cache configuration. We compute energy_cache_access and energy_cache_block_refill using Cacti 3.2 for both L1 and two caches.

The energy_next_level_mem includes the energy used accessing the L2 cache (if present) and the energy of accessing off-chip memory. The energy_static 


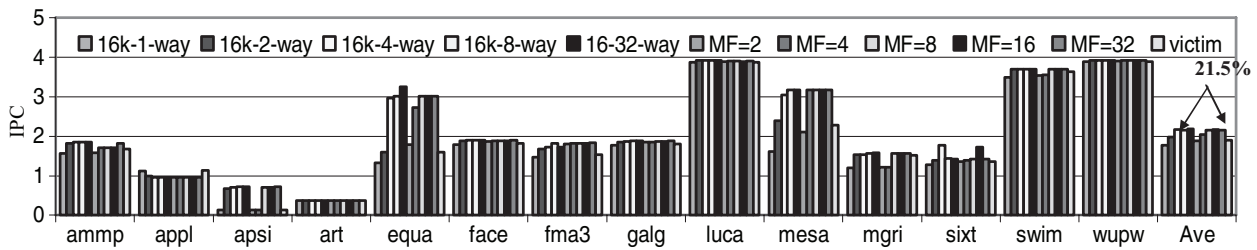

Fig. 14. IPC of a direct-mapped, 2-, 4-, 8-, and 32-way, the B-Cache, and a 16-entry victim buffer at cache size of $16 \mathrm{kB}$ (only the first four letters of the benchmarks are shown to save space).

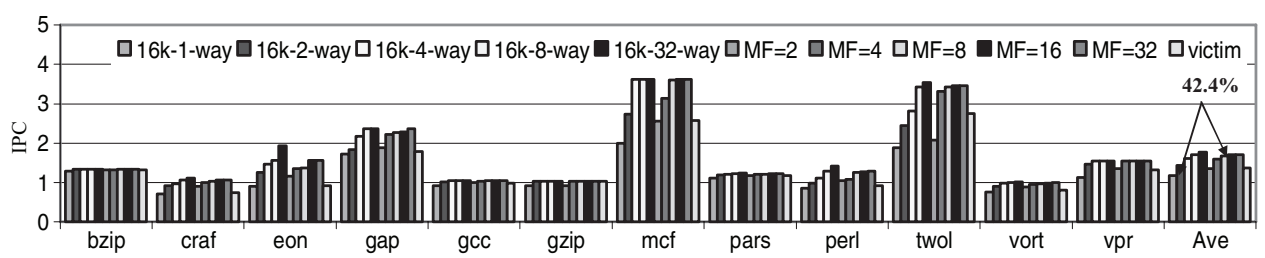

Fig. 15. IPC of a direct-mapped, 2-, 4-, 8-, and 32-way, the B-Cache, and a 16-entry victim buffer at cache size of $16 \mathrm{kB}$ (only the first four letters of the benchmarks are shown to save space).

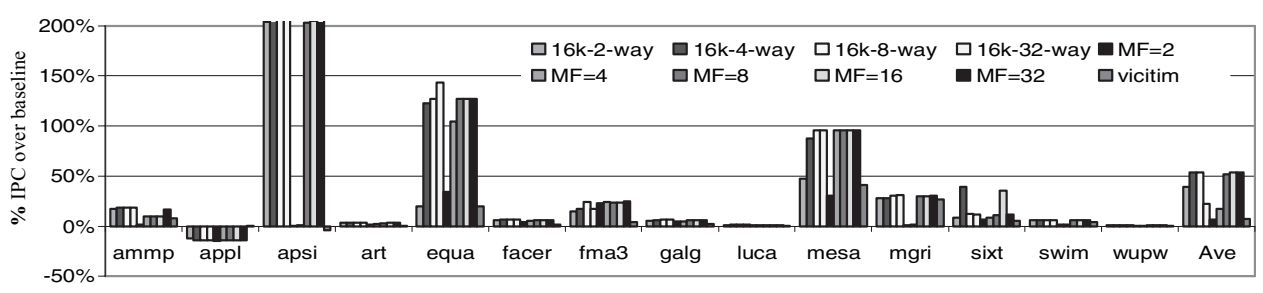

Fig. 16. IPC improvements of a 2-, 4-, 8-, and 32-way, the B-Cache, and a 16-entry victim buffer at cache size of $16 \mathrm{kB}$ (only the first four letters of the benchmarks are shown to save space).

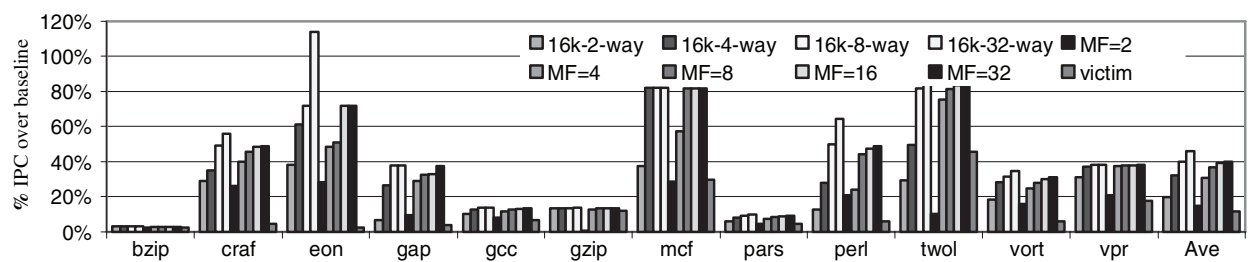

Fig. 17. IPC improvements of a 2-, 4-, 8-, and 32-way, the B-Cache, and a 16-entry victim buffer over the baseline at cache size of $16 \mathrm{kB}$ (only the first four letters of the benchmarks are shown to save space).

per_cycle is the total static energy consumed per cycle. Both the energy used accessing off chip memory and energy_static_per_cycle are highly system dependent. Using a methodology similar to [Zhang et al. 2003], we evaluate the energy of accessing the off-chip memory as 100 times larger than the baseline.

We evaluate the static energy as 50\% ( $k_{-}$static) of the total energy that includes both dynamic and static energy. Figure 19 shows the energy of 2-, 4-, and 8-way, the $B$-Cache, and the victim buffer normalized to the baseline. On average, the $B$-Cache consumes the least amount of energy and is $2 \%$ less than 


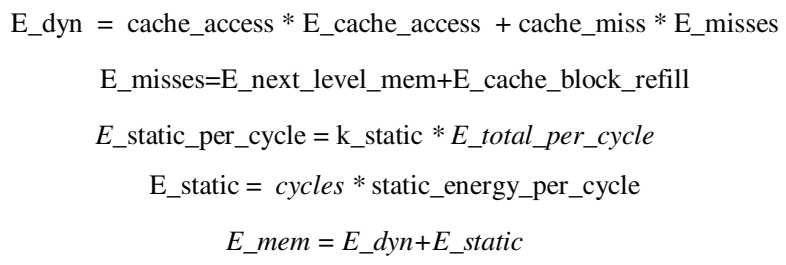

Fig. 18. Equations for energy evaluation.

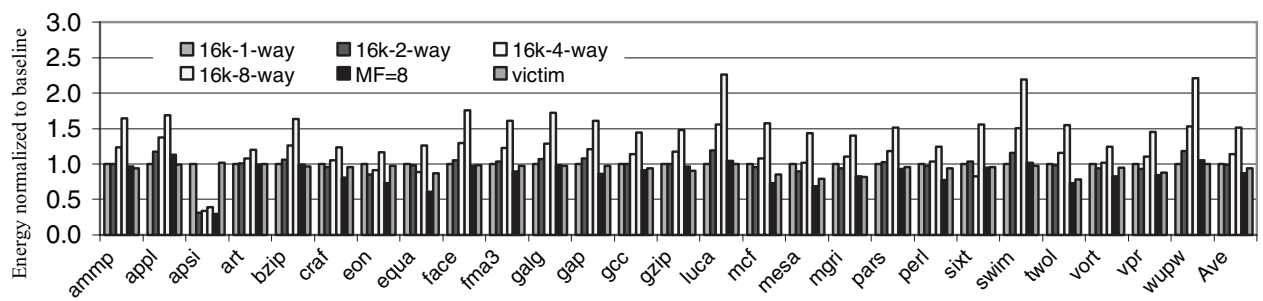

Fig. 19. Normalized overall energy of a 1-, 2-, 4-, and 8-way, the B-Cache, and a 16-entry victim buffer over the baseline at a cache size of $16 \mathrm{kB}$ (only the first four letters of the benchmarks are shown to save space).

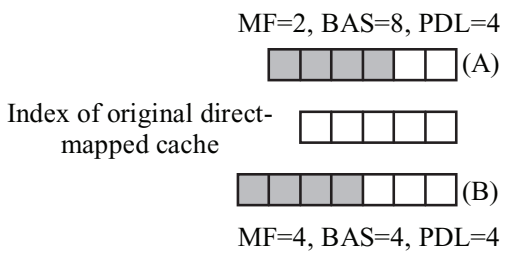

Fig. 20. Tradeoff of MF, BAS, and PDL (programmable decoder length).

the baseline. The greatest energy reduction is seen in crafty; where the energy is reduced by $14 \%$. The $B$-Cache reduces the miss rate and, hence, the accesses to the second-level cache, which is more power costly. During a cache miss, the $B$-Cache also reduces the cache memory accesses through the miss prediction of the programmable decoder, which makes the effective power overhead much less. In Section 6.3, we show that the programmable decoder can predict, on average, around $80 \%$ of the cache misses, thus reducing the power to access both the data and tag during a cache miss.

\subsection{Design Tradeoffs for MP and BAS for a Fixed Length of Programmable Decoder}

For a fixed length of programmable decoder, such as $\mathrm{PI}=4$, the $B$-Cache has two design options, as shown in Figure 20. In design A, the B-Cache uses MF $=2$ and $\mathrm{BAS}=8$. In design $\mathrm{B}$, the $B$-Cache uses $\mathrm{MF}=4$ and $\mathrm{BAS}=4$. The question becomes a matter of which design has a higher miss rate reduction. In design A, the $B$-Cache has eight clusters and the miss rate reduction would approach that of an 8-way cache. However, $\mathrm{MF}=2$ means the programmable decoder hit rate is high and the $B$-Cache cannot fully exploit the replacement policy. 
Table VII. The Miss Rate Reductions (\%) of the B-Cache Compared with a Direct-mapped Cache at Varied $M F, B A S$, and Programmable Decoder Length (PDL)

\begin{tabular}{|c|c|c|c|c|c|c|c|c|}
\hline \multirow{2}{*}{} & \multicolumn{5}{|c|}{ Instruction cache } & \multicolumn{4}{c|}{ Data cache } \\
\cline { 2 - 9 } & $\mathrm{MF}=2$ & $\mathrm{MF}=4$ & $\mathrm{MF}=8$ & $\mathrm{MF}=16$ & $\mathrm{MF}=2$ & $\mathrm{MF}=4$ & $\mathrm{MF}=8$ & $\mathrm{MF}=16$ \\
\hline $\mathrm{BAS}=8$ & 26.2 & 49.1 & 54.1 & 57.4 & 11.4 & 15.4 & 23.5 & 24.2 \\
\hline $\mathrm{BAS}=4$ & 42.4 & 46.6 & 49.3 & 49.7 & 14.5 & 21.2 & 21.8 & 22 \\
\hline $\mathrm{PDL}$ & 3 & 4 & 5 & 6 & 3 & 4 & 5 & 6 \\
\hline
\end{tabular}

Table VIII. The IPCs of a Processor with B-Cache at Varied MF, BAS, and Programmable Decoder Length (PDL)

\begin{tabular}{|c|c|c|c|c|c|c|c|c|}
\hline & \multicolumn{4}{|c|}{ Integer benchmarks } & \multicolumn{3}{c|}{ Floating-point benchmarks } \\
\cline { 2 - 9 } & $\mathrm{MF}=2$ & $\mathrm{MF}=4$ & $\mathrm{MF}=8$ & $\mathrm{MF}=16$ & $\mathrm{MF}=2$ & $\mathrm{MF}=4$ & $\mathrm{MF}=8$ & $\mathrm{MF}=16$ \\
\hline $\mathrm{BAS}=8$ & 1.36 & 1.60 & 1.68 & 1.70 & 1.88 & 2.05 & 2.15 & 2.17 \\
\hline $\mathrm{BAS}=4$ & 1.52 & 1.59 & 1.60 & 1.61 & 2.02 & 2.12 & 2.14 & 2.14 \\
\hline $\mathrm{PDL}$ & 3 & 4 & 5 & 6 & 3 & 4 & 5 & 6 \\
\hline
\end{tabular}

Table IX. Programmable Decoder Hit Rate (\%) During Cache Misses of the B-Cache at Varied $M F$, $B A S$, and Programmable Decoder Length (PDL)

\begin{tabular}{|c|c|c|c|c|c|c|c|c|}
\hline & \multicolumn{4}{|c|}{ Instruction cache } & \multicolumn{4}{|c|}{ Data cache } \\
\hline & $\mathrm{MF}=2$ & $\mathrm{MF}=4$ & $M F=8$ & $M F=16$ & $\mathrm{MF}=2$ & $\mathrm{MF}=4$ & $\mathrm{MF}=8$ & $M F=16$ \\
\hline $\mathrm{BAS}=8$ & 63.0 & 19.0 & 8.4 & 5.2 & 53.6 & 26.6 & 10.3 & 5.5 \\
\hline $\mathrm{BAS}=4$ & 27.1 & -11.1 & $7.7 \mathrm{~d}$ & 0.7 & 32.2 & 12.6 & 7.9 & ( 5.4 \\
\hline PDL & $\begin{array}{l}1 \\
3\end{array}$ & $\frac{1}{4}$ & $\frac{1}{5}$ & $\begin{array}{l}t \\
6\end{array}$ & $\begin{array}{l}7 \\
3\end{array}$ & $\begin{array}{l}t \\
4\end{array}$ & 5 & $\begin{array}{l}1 \\
6\end{array}$ \\
\hline
\end{tabular}

At this point, the miss rate reduction may be low. Tables VII, VIII, and IX show the miss rate reduction, IPCs, and programmable decoder hit rate of the $B$-Cache at varied MF,BAS, and programmable decoder combinations. For the same programmable decoder length, the miss rate reduction of the design $B$ $(\mathrm{BAS}=4)$ outperforms the design $\mathrm{A}(\mathrm{BAS}=8)$ when the programmable decoder is less than six.

This is because the programmable decoder hit rate of design $B$ is lower than design A, since design B has a larger MF. However, when the programmable decoder is increased to six and its hit rates are low for both designs, and the effect of high clusters appears to be important, then design A has a higher miss reduction rate than design B. Based on our timing analysis in Section 5.1, we can use 
a 6-bit programmable decoder without incurring access time overhead; therefore, we design the $B$-Cache with $\mathrm{MF}=8$ and $\mathrm{BAS}=8$. This result also presents design options for varied programmable decoder values and corresponding miss rate reductions.

\subsection{Balance Evaluation}

We classify a "set" as frequent hit or frequent miss sets when the cache hits or misses occurring in a set are two times higher than the average. We classify a set as less accessed when the total accesses to a set are less than one-half of the average. We measure the frequent hit, miss, and less accessed sets of the original direct-mapped and the $B$-Cache and show the results in Tables $\mathrm{X}$ and $\mathrm{XI}$ for the baseline instruction and data caches.

Comparing the $B$-Cache with the baseline of a data cache, we find the number of frequent hit sets is changed from 7.0 to $10.6 \%$, but accounts for $44.8 \%$ of the total hits instead of $66.8 \%$. This represents a $22.0 \%$ reduction, which means the cache hits occur across more sets. The frequent miss sets are reduced from 5.1 to $2.8 \%$ and the total misses are also reduced from 37.9 to $9.5 \%$, explaining the cache miss reductions of the $B$-Cache. The less accessed sets are reduced from 63.9 to $38.3 \%$, which means more cache sets are efficiently used to accommodate the cache accesses. Similar situations exist for the instruction cache.

From Figures 8 and 9, we observe that the miss rates of some benchmarks, such as art and gzip, are high and reduction is very limited for both the setassociative caches and the B-Cache. From Table X, we observe that there are very limited frequent miss sets for these benchmarks, which means the cache misses occur evenly on all cache sets. Continuing to balance the accesses to the sets would not bring significant miss rate reductions. For other benchmarks, such as parser, the miss rate reductions are higher than $40 \%$.

The frequent miss sets are reduced from 5.3 to $0.0 \%$; however, the total cache misses occurring on the frequent miss sets are reduced from 25.7 to $0.0 \%$, which means the conflict addresses have been largely remapped to less accessed sets, thus reducing the cache misses.

The $B$-Cache still has many cache sets not being accessed. This is because we try to minimize the cache misses and remap missed addresses to less accessed

sets. On average, the cache miss rate is less than 1 and $10 \%$ for the instruction and data cache, respectively. Even if we remap all the cache misses to less accessed sets, they are still accessed more infrequently than the frequent hit sets. Based on the above discussion, we can see that other cache design techniques, such as Drowsy cache [Flautner et al. 2002] and Cache decay [Kaxiras et al. 2001] that take advantage of the nonuniform cache accesses, can still be used on the $B$-Cache, since these less accessed sets can still be in a drowsy state.

\subsection{The Effect of L1 Cache Sizes}

The miss rate reductions of twelve cache configurations are shown in Figure 21. It includes the $B$-Cache at a memory-mapping factor $\mathrm{MF}=2,4,8$, and 16 , with the $B$-Cache associativity BAS $=4$ and 8 . The conventional 2-, 4-, and 8-way caches, at sizes of 8 and $32 \mathrm{kB}$, and a 16-entry victim buffer are also reported. 


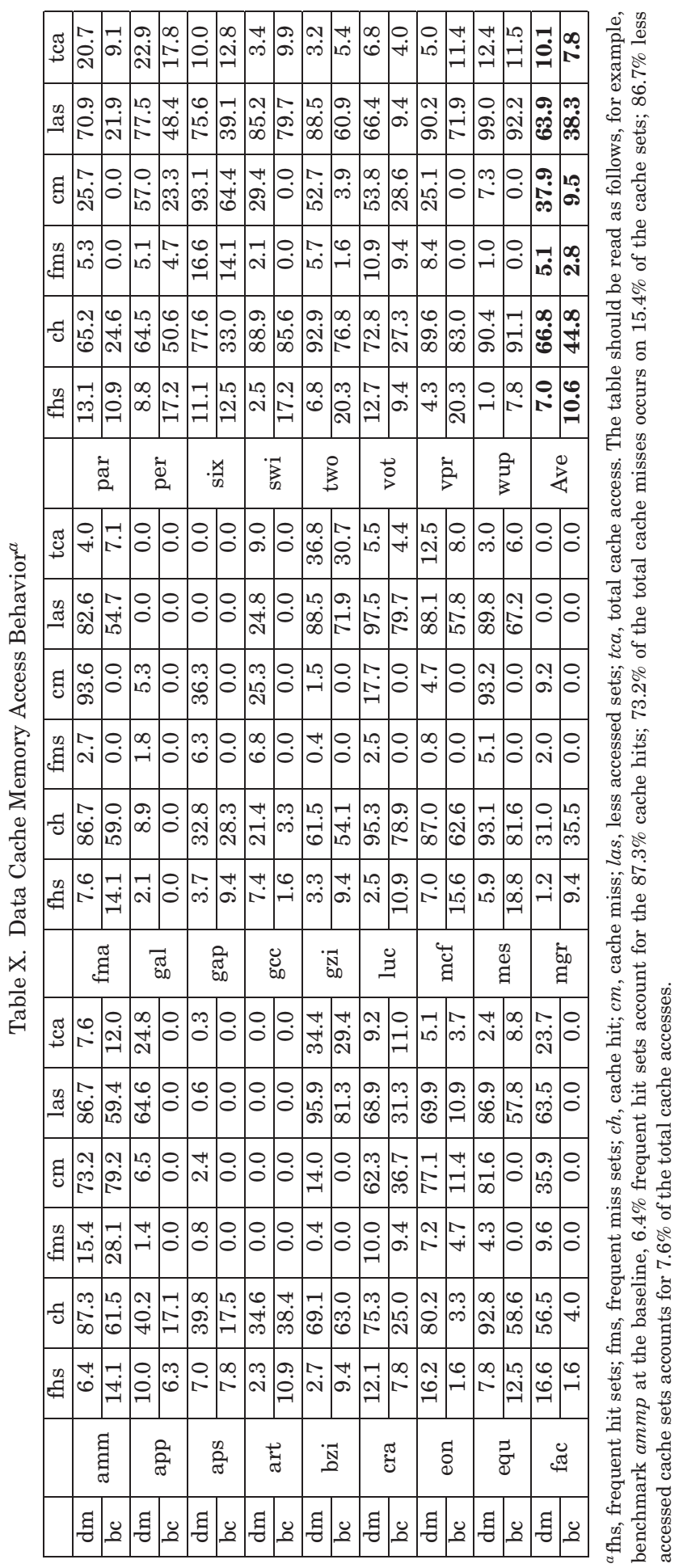




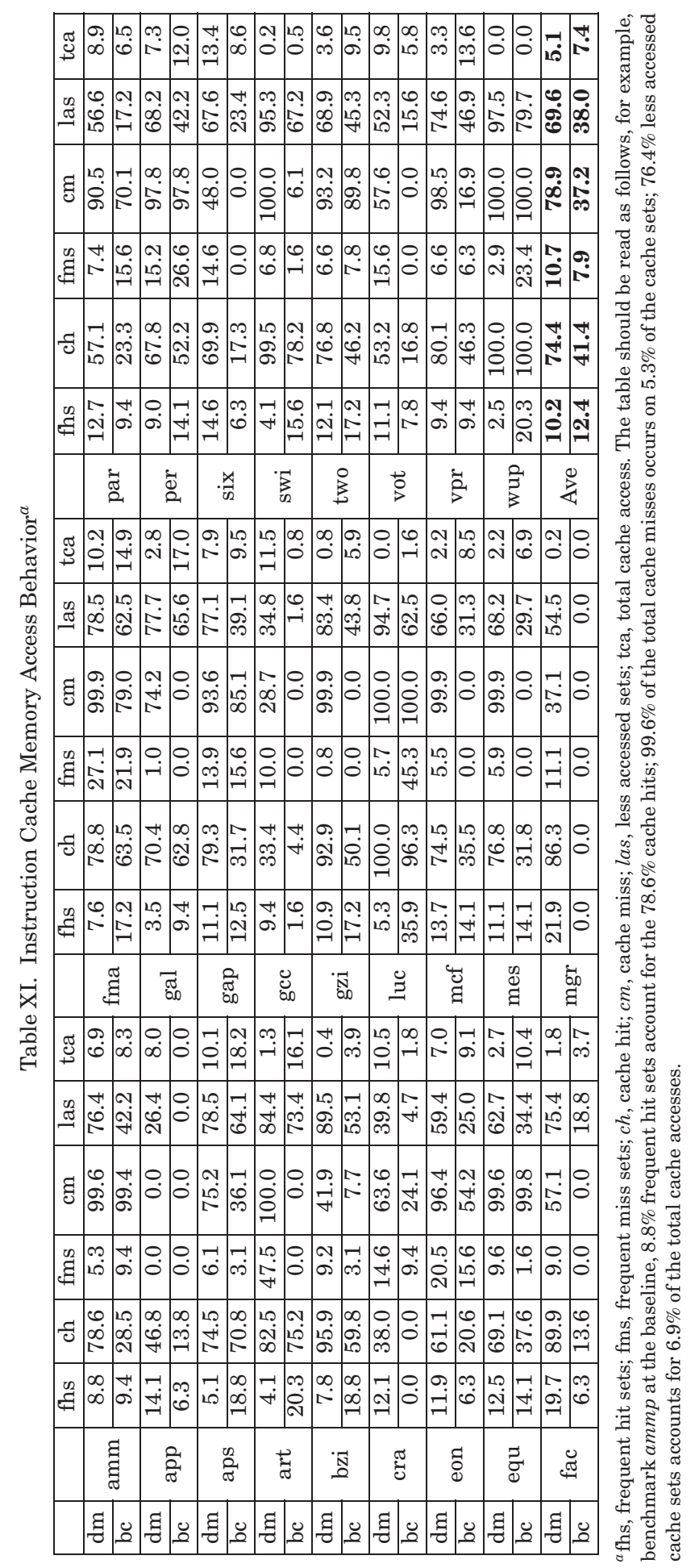

ACM Transactions on Architecture and Code Optimization, Vol. 4, No. 4, Article 24, Publication date: January 2008. 


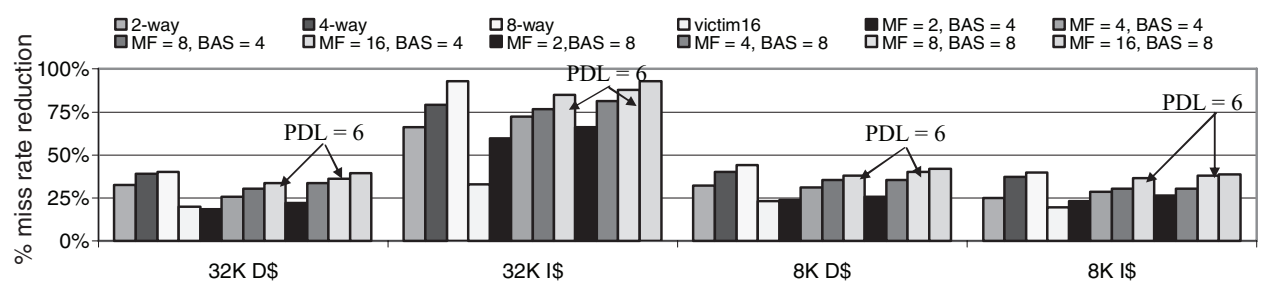

Fig. 21. Miss rate reductions of the B-Cache at cache sizes of 32 and $8 \mathrm{kB}$.

From Figure 21, we can tell the $B$-Cache exhibits similar miss rate reductions at sizes of 8 and $32 \mathrm{kB}$ compared to the $16 \mathrm{kB}$ direct-mapped caches. The miss rate reductions increase when the MF is increased from 2 to 16 when the BAS are 4 and 8 . For the same programmable decoder length of 6 bits, the B-Cache at $\mathrm{MF}=8$ and $\mathrm{BAS}=8$ has a higher miss rate reduction than the design of $\mathrm{MF}=16$ and $\mathrm{BAS}=4$. Therefore, we conclude that for the $B$-Cache, the design with $\mathrm{MF}=8$ and $\mathrm{BAS}=8$ is the best for the cache sizes of 8,16 , and $32 \mathrm{kB}$.

\subsection{Comparison with a Victim Buffer}

We compare the $B$-Cache with a 16-entry victim buffer with a line size of 32 bytes. A victim buffer with more than 16 entries may not bring significant miss rate reduction, but may increase the buffer's access time and energy. The miss rate reductions of the buffer for both instruction and data cache are shown in Figures 7, 8, 9, 10, and 21 at cache sizes of 8, 16, and $32 \mathrm{kB}$. The average instruction miss rate reduction of the $B$-Cache is 20.0 and $40.7 \%$ higher than the victim buffer for the CINT2K and CFP2K, respectively. For the data cache, the miss rate reduction of the $B$-Cache is 17.9 and $25.7 \%$ higher than the victim buffer. There is only one benchmark, applu, which is the miss rate reduction of the $B$-Cache that is lower than the victim buffer. On average, the miss rate reduction of the $B$-Cache is 15.4 and $42.3 \%$ higher than the victim buffer for the $32 \mathrm{kB}$ data and instruction caches, respectively. For the $8 \mathrm{kB}$ data and instruction caches, the miss rate reduction of the B-Cache is 16.2 and $19.3 \%$ higher than the victim buffer, respectively.

\subsection{Comparison with a Highly Associative Cache}

The highly associative cache (HAC) [Santhanam et al. 1998] has been proposed for low-power embedded systems. To reduce the power consumption, the HAC has been aggressively partitioned with a subarray size of $1 \mathrm{kB}$. Only one subarray of the HAC is accessed to reduce the power consumption. Therefore, the search of the CAM tags has to wait for the completion of the global decoding, which prolongs the access time of the HAC. In fact, the HAC is an extreme case of the $B$-Cache, where the decoder of the HAC is fully programmable. For a $16-\mathrm{kB}$ HAC with a line size of 32 bytes and an associativity of 32 , the CAM tag (also the programmable decoder) length of the HAC is $23+3$ (status) $=26$ bits, while the $B$-Cache uses 6 bits of CAM to achieve similar miss rate reductions. Therefore, the HAC can be improved using the technique we propose to reduce both the power consumption and area of the CAM. 


\subsection{Issues on Virtually/Physically Addressed Tagged Caches}

Our scheme requires the decoder to be 3 bits longer than the baseline. The extra 3 bits come from the tag bits of the original design. These 3-bit tags are required no later than the set index. If the tag, but not the set index, needs to be first translated by a translation lookaside buffer (TLB), this presents a problem, since the programmable index lookup cannot proceed. Such situations occur in a virtually indexed and physically tagged (V/P) cache, such as in the PowerPC [IBM]. Similar problems exist for the skewed-associative [Seznec 1993] and the way-halting cache [Zhang et al. 2005], where the least 4 bits of the virtual tags from the processor are equal to the least 4 bits of the physical tags stored in the cache tags, which means those tag bits do not need translations. For the $B$-Cache, only the least 3 bits of the tag are required to be the same as those stored in the tag memory. We may treat these 3 bits as virtual indexes. The $B$-Cache would work under other tag and data array addressing using either the virtual or the physical address, such as virtually indexed, virtually tagged; physically indexed, virtually tagged; and physically indexed, physically tagged caches.

\section{RELATED WORK}

The related work can be generally categorized into two types. One type is to reduce the miss rate of direct-mapped caches. The other type is to reduce the access time of set-associative caches.

\subsection{Reducing Miss Rate of Direct-mapped Caches}

Techniques to resolve misses of direct-mapped caches have been proposed with the help of operating systems. Page allocation [Bershad et al. 1994] can be optimized to reduce the misses of a direct-mapped cache with an operating system involved. A cache miss lookaside buffer is used to detect the cache misses by recording a history of misses. A software policy implemented in the operating system removes the misses by dynamically re-mapping pages whenever large numbers of misses are detected. This technique enables a direct-mapped cache to perform nearly as well as a 2 -way set-associative cache. The $B$-Cache is implemented entirely in hardware with as low a miss rate as a 4 -way cache.

The column-associative cache [Agarwal and Pudar 1993] uses a directmapped cache and an extra bit for dynamically selecting alternate hashing functions. This design improves the miss rate to a 2-way cache at a cost of an extra rehash bit and a multiplexer (for the address generation) that could affect the critical time of the cache hit. Column-associative cache can be extended to include multiple alternative locations, which are described in Chung and PMF [1998] and Zhang et al. [1997]. Peir et al. [1998] attempts to use cache space intelligently by taking advantage of the cache holes during the execution of a program. They proposed an adaptive group-associative cache (AGAC) that can dynamically allocate the data to the cache holes and, therefore, reduce the misses of a direct-mapped cache. Both the AGAC and the B-Cache can achieve comparable miss rate reductions to a 4-way cache. However, the AGAC needs three cycles to access these relocated cache lines, which account for $5.24 \%$ of 
the total cache hits, while the $B$-Cache needs one cycle for all cache hits. The skewed-associative cache [Seznec 1993] is a 2-way cache that exploits two or more indexing functions derived by XORing two $m$-bit fields from an address to generate an $m$-bit cache index to achieve the miss rate to that of a same-sized 4-way cache.

\subsection{Reducing Access Time of Set-associative Caches}

Partial address matching [Liu 1994] reduces the access time of set-associative caches by predicting the hit way. The tag memory is separated into two arrays main directory (MD) and partial address directory (PAD). The PAD contains only a small part of full tag bits (e.g., 5 bits); therefore, the comparison of PAD is faster than a full tag comparison. The results of the $\mathrm{PAD}$ comparison can be used to predict the hit way, while the result of the MD comparison verifies the hit. If the PAD prediction is not correct, a second cycle is required to access the correct way. The difference-bit cache [Juan et al. 1996] is a 2-way set-associative cache with an access time close to a direct-mapped cache. The difference bit in two tags is dynamically determined by using a special decoder and used to select the potential hit way from the two ways of the 2-way set-associative cache.

Compared with the partial address matching method, our technique does not require the extra cycle to fetch the desired data because of the miss prediction in the PAD comparison. The access time of the difference bit cache is slower than the $B$-Cache. Since the difference-bit cache is used in a 4-way cache, the area and access time will be significantly increased compared to a direct-mapped cache [Juna et al. 1996]. Furthermore, the B-Cache can achieve a miss rate as low as a traditional 4-way cache, while the difference bit-cache [Juan et al. 1996] cannot be better than a 2 -way cache.

The concept of partial address matching has also been exploited in inexpensive implementations of the set-associative caches to reduce the complexity of L2 set-associative cache designs [Kessler et al. 1989]. The inexpensive associative cache scans tags in sequence from most used to least recently used or partially compares a few bits from each tag to reduce the number of tags that must be examined sequentially. These techniques increase the access time of the cache, so that the inexpensive caches are used for L2 cache designs.

Compared with the previous techniques, the B-Cache can be applied to both high performance and low-power embedded systems. The mapping of addresses to cache sets is automatically and dynamically balanced by the cache controller without any software intervention. In practice, our technique is more feasible and can be easily implemented, because the $B$-Cache does not need any counters or tables to collect cache access information on the fly or special algorithms that dynamically control the caching. Our technique is more powerful and promising, since the $B$-Cache would not disrupt the cache pipeline that is generally exploited in modern high-performance processors.

\section{CONCLUSIONS}

Memory accesses are not uniformly distributed in a program execution, creating an imbalance in the demand on individual cache sets. We have proposed the 
$B$-Cache, a design that allows access to cache sets to be balanced by increasing the decoder length and incorporating a replacement policy to the direct-mapped cache. We have also proposed programmable decoders to dynamically determine which memory address has a mapping to the cache set. We expect the proposed programmable decoders can also be used in L2 cache designs to balance the cache set access and reduce cache misses.

\section{REFERENCES}

Agarwal, A. And Pudar, S. D. 1993. Column-associative caches: A technique for reducing the miss rate of direct-mapped caches. In Proceedings of the International Symposium on Computer Architecture. 179-180.

Agarwal, V., Hrishikesh, M. S., Keckler, S. W., And Burger, D. 2000. Clock rate versus IPC: The end of the road for conventional microarchitectures. In Proceedings of the 27th International Symposium on Computer Architecture (June). 248-259.

Agarwal, A., Roy, K., and ViJaykumar, T. N. 2003. Exploring high bandwidth pipelined cache architecture for scaled technology. In Proceedings of Design, Automation, and Test in Europe Conference and Exhibition.

Bershad, B. N., Lee, D., and Chen, J. B. 1994. Avoiding conflict misses dynamically in large direct-mapped caches. In Proceedings of the Architecture Support for Programming Language and Operating Systems.

Burger, D. And Austin, T. M. 1997. The SimpleScalar Tool Set, Version 2.0. Univ. of WisconsinMadison Computer Sciences Dept. Technical Report \#1342, June.

Cadence Corporation. http://www.cadence.com

Calder, B., Grunwald, D., And Emer, J. 1996. Predictive sequential associative cache. In Proceedings of the High Performance Computer Architecture. 244-253.

Chung, B. And Pmf, J. 1998. LRU-based column associative caches. Camp. Arch. News 26, 2, 9-17.

Easton, M. C. and Fagin, R. 1978. Cold-start vs. warm start miss ratios. Commun. ACM 21, 10, $866-872$.

Eғтнymio, A. And Garside, J. 2002. An adaptive serial-parallel CAM architecture for low-power cache blocks. In Proceedings of International Symposium of Low Power Electronics and Design.

Flautner, K., Kim, N. S., Martin, S., Blaauw, D., and Mudge, T. 2002. Drowsy caches: Simple techniques for reducing leakage power. In Proceedings of the International Symposium on Computer Architecture.

Ghose, K. And Kamble, M. B. 1999. Reducing power in superscalar processor caches using subbanking, multiple line buffers, and bitline segmentation. In Proceedings of IEEE International Symposium on Low Power Electronics and Design.

Givargis, T. 2003. Improved indexing for cache miss reduction in embedded systems. In Proceedings of Design Automation Conference.

JoupPI, N. P. 1990. Improving direct-mapped cache performance by the addition of a small fully-associative cache and prefetch buffers. In Proceedings of IEEE International Symposium on Computer Architecture (May). 364-373.

Juan, T., Lang, T., and Navarro, J. 1996. The difference-bit cache. In Proceedings of the 27th International Symposium on Computer Architecture.

Kaxiras, S., Hu, Z., And Martonosi, M. 2001. Cache decay: Exploiting generational behavior to reduce cache leakage power. In Proceedings of International Symposium on Computer Architecture.

Kharbutli, M., Irwin, K., Solihin, Y., and Lee, J. 2004. Using prime numbers for cache indexing to eliminate conflict misses. In Proceedings of the 10th IEEE International Symposium on High Performance Computer Architecture. 288-299.

Kessler, R. E., Jooss, R., Lebeck, A., And Hill, M. D. $1989 . \quad$ Inexpensive implementations of set-associativity. In Proceedings of the 16th Annual International Symposium on Computer Architecture, Jerusalem, Israel (Apr.). 131-139.

Liv, L. 1994. Cache design with partial address matching. In Proceedings of the International Symposium on Microarchitecture.

ACM Transactions on Architecture and Code Optimization, Vol. 4, No. 4, Article 24, Publication date: January 2008. 
Naffziger, S. D., Colon-Bonet, G., Fischer, T., Riedlinger, R., Sullivan, T. J., and Grutkowski, T. 2002. The implementation of the Itanium 2 microprocessor. IEEE Journal of Solid-State Circuits $37,11$.

PeIR, J., Hsu, W., Young, H., AND ONG, S. 1996. Improving cache performance with balanced tag and data paths. In Proceedings of the 7th International Conference Architectural Support for Programming Languages and Operating Systems. 268-278.

PeIR, J., LeE, Y., AND Hsu, W. 1998. Capturing dynamic memory reference behavior with adaptive cache topology. In Proceedings of the 8th International Conference on Architectural Support for Programming Language and Operating Systems. 240-250.

Reinmann, G. And Jouppi, N. P. 1999. CACTI2.0: An Integrated Cache Timing and Power Model. COMPAQ Western Research Lab.

SANTHANAM, S. ET AL. 1998. A low-cost, 300-MHz, RISC CPU with attached media processor. IEEE Journal of Solid-State Circuits 33, 11, 1829-1839.

SEznec, A. 1993. A case for two-way skewed-associative caches. In Proceedings of the International Symposium on Computer Architecture.

Sherwood, T., Perelman, E., Hamerly, G., and Calder, B. 2002. Automatically characterizing large scale program behavior. In Proceedings of the 10th International Conference on Architectural Support for Programming Languages and Operating Systems.

Standard Performance Evaluation Corporation. http://www.specbench.org/osg/cpu2000/.

Sun Microsystems, Inc. 2006. http://www.sun.com/servers/entry/v210/datasheet.pdf.

Weaver, C. T. Pre-compiled SPEC2000 Alpha Binaries. Available at: http://www.simplescalar.org.

WiLkEs, M. V. 1995. The memory wall and the CMOS end-point. ACM Computer Architecture News 23,4 .

Wulf, W. A. ANd McKeE, S. A. 1995. Hitting the memory wall, implications of the obvious. ACM Computer Architecture News 23, 1.

Yoshimoto, M. ет AL. 1983. A divided word-line structure in the static RAM and its application to a 64k full CMOS RAM. IEEE J. Solid-State Circuits SC-21, 479-485.

ZhANG, C. 2006. Balanced cache: Reducing conflict misses of direct-mapped caches through programmable decoders. In Proceedings of the International Symposium on Computer Architecture.

ZHANG, C., VAHID, F., AND NAJJAR, W. 2003. A highly-configurable cache architecture for low power embedded systems. In Proceedings of the International Symposium on Computer Architecture.

Zhang, C., VAhID, F., YANG, J., AND NAJJAR, W. 2005. A way-halting cache for low-energy highperformance systems. ACM Transactions on Architecture and Code Optimization (TACO) 2, 1, $34-54$.

Zhang, C., Zhang, X., And Yan, Y. 1997. Two fast and high-associativity cache schemes. IEEE Micro 17, 5, 40-49.

Received August 2006; revised March 2007; accepted May 2007 